May 5, 2014

\title{
Structure and Dynamics of the Accretion Process and Wind in TW Hya ${ }^{1}$
}

\author{
A. K. Dupree, N. S. Brickhouse, S. R. Cranmer, P. Berlind ${ }^{2}$, Jay Strader ${ }^{3}$ \\ Harvard-Smithsonian Center for Astrophysics, Cambridge, MA 02138, USA \\ Graeme H. Smith \\ University of California Observatories/Lick Observatory, University of California, \\ Santa Cruz, CA 95064
}

\begin{abstract}
Time-domain spectroscopy of the classical accreting T Tauri star, TW Hya, covering a decade and spanning the far UV to the near-infrared spectral regions can identify the radiation sources, the atmospheric structure produced by accretion, and properties of the stellar wind. On time scales from days to years, substantial changes occur in emission line profiles and line strengths. Our extensive time-domain spectroscopy suggests that the broad near-IR, optical, and far-uv emission lines, centered on the star, originate in a turbulent post-shock region and can undergo scattering by the overlying stellar wind as well as some absorption from infalling material. Stable absorption features appear in $\mathrm{H} \alpha$, apparently caused by an accreting column silhouetted in the stellar wind. Inflow of material onto the star is revealed by the near-IR He I $10830 \AA$ line, and its free-fall velocity correlates inversely with the strength of the post-shock emission, consistent with a dipole accretion model. However, the predictions of hydrogen line profiles based on accretion stream models are not well-matched by these observations. Evidence of an accelerating warm to hot stellar wind is shown by the near-IR He I line, and emission profiles of C II, C III, C IV, N V, and O VI. The outflow of material changes substantially in both speed and opacity in the yearly sampling of the near-IR He I line over a decade. Terminal outflow velocities that
\end{abstract}

\footnotetext{
${ }^{2}$ Fred L. Whipple Observatory, Amado, AZ

${ }^{3}$ Michigan State University, East Lansing, MI
} 
range from $200 \mathrm{~km} \mathrm{~s}^{-1}$ to almost $400 \mathrm{~km} \mathrm{~s}^{-1}$ in He I appear to be directly related to the amount of post-shock emission, giving evidence for an accretion-driven stellar wind. Calculations of the emission from realistic post-shock regions are needed.

Subject headings: stars: individual (TW Hydrae) - stars:pre-main sequence stars: variables: T Tauri, Herbig Ae/Be - stars: winds, outflows - Accretion, accretion disks

\section{Introduction}

TW Hya (CD -34 7151, TWA 1, HIP 53911) remains arguably the closest accreting T Tauri Star (Wichmann et al. 1998), and is oriented with its rotation axis almost along our line of sight which sets the surrounding accretion disk approximately in the plane of the sky (Krist et al. 2000; Qi et al. 2004). These characteristics make TW Hya a subject of intensive study at all wavelengths because it is bright and the accretion process is directly accessible by this polar orientation (Donati et al. 2011; Johnstone et al. 2014).

TW Hya has been a frequent target of intensive study of optical emission lines along with several other bright T Tauri stars: BP Tau (Gullbring et al. 1996); DF Tau (Johns-Krull \& Basri 1997); DQ Tau (Basri et al. 1997); DR Tau (Alencar et al. 2001); and RW Aur (Alencar et al. 2005) among them. These studies reveal the intrinsic variability of the optical lines. These stars have larger inclinations than TW Hya, $\left[20^{\circ}\right.$ for DR Tau (Schegerer et al. 2009); $39^{\circ}$ for BP Tau (Guilloteau et al. 2011); the star RW Aur A is inclined by $37^{\circ}$; others are not known]. The uniqueness of TW Hya resides in the imaging of the surrounding disk

\footnotetext{
${ }^{1}$ Data presented herein were obtained at the W. M. Keck Observatory, which is operated as a scientific partnership among the California Institute of Technology, the University of California, and the National Aeronautics and Space Administration. The Observatory was made possible by the generous financial support of the W. M. Keck Foundation. Infrared spectra were taken at the Gemini Observatory, which is operated by the Association of Universities for Research in Astronomy, Inc., under a cooperative agreement with the NSF on behalf of the Gemini partnership: the National Science Foundation (United States), formerly the Science and Technology Facilities Council (United Kingdom), the National Research Council (Canada), CONICYT (Chile), the Australian Research Council (Australia), Ministério da Ciência e Tecnologia (Brazil) and Ministerio de Ciencia, Tecnología e Innovación Productiva (Argentina). This paper also includes spectra gathered with the 6.5-meter Magellan Telescope/CLAY located at Las Campanas Observatory, Chile. Additional spectra were obtained at the $1.5 \mathrm{~m}$ Tillinghast Telescope at the Fred Lawrence Whipple Observatory of the Smithsonian Astrophysical Observatory.
} 
in the infrared (Krist et al. 2000) and in the sub-millimeter range (Qi et al. 2004) revealing its face-on orientation. Thus the interesting polar region where accretion is ongoing can be viewed directly. This paper reports spectral sequences not only from the optical region, but the near-infrared and ultraviolet as well. The broad wavelength coverage spans accretion phenomena, the presence of winds, and the intrinsic chromosphere/corona of the star.

TW Hya itself is of low mass. Estimates range from $0.4-0.8 \mathrm{M}_{\odot}$ (Batalha et al. 2002; Donati et al. 2011; Vacca \& Sandell 2011). The spectral type is believed to be close to a K7 dwarf (Alencar \& Batalha 2002). A possible later spectral type (M2.5V, Vacca \& Sandell 2011) is controversial (Andrews et al. 2012; McClure et al. 2013). The inferred mass and radius for a $\mathrm{M} 2.5 \mathrm{~V}$ star lead to lower free-fall accretion velocities and consequently shock temperatures lower than measured directly (Brickhouse et al. 2010). This star has become a fiducial object in the astrophysics of accretion and low mass star formation.

Currently, the 'standard model' of accretion suggests (Hartmann 1998) that material from a surrounding disk is channeled by magnetic fields in an 'accretion funnel' towards the central star forming an accretion shock, a small post-shock cooling zone, and a hot spot or ring on or near the stellar surface. The source of optical emission is assigned to the accreting funnel flows (Hartmann et al. 1994; Muzerolle et al. 2001; Kurosawa \& Romanova 2013). Alencar and Batalha (2002) in a very detailed analysis of 42 optical spectra of TW Hya acquired mostly nightly over a 1.4 year period described the behavior of several emission lines: $\mathrm{H} \alpha, \mathrm{H} \beta$, He I ( $\lambda 5876)$ and $\mathrm{Na} \mathrm{D}$. They noted both outflow and inflow signatures in these profiles and documented a correlation between veiling corrected equivalent widths of major lines and veiling. This suggests that increased veiling - believed to arise from the continuum and lines (Gahm et al. 2008) produced by the accretion hot spot (or ring) - is related to the increased equivalent width of optical emission lines. The accretion rate and its variation can be inferred directly from the Ne IX lines in the X-ray spectrum (Brickhouse et al. 2012). However, recent optical spectroscopy simultaneous with X-ray measurements demonstrated the progression of accreted material in the post-shock cooling zone through the stellar atmosphere, producing optical emission, followed by the heating of the photosphere, and subsequent enhancement of the corona (Dupree et al. 2012). This sequence challenges the 'standard model' which attributes optical emission to the accretion funnels (Muzerolle et al. 2001, 2005; Natta et al. 2004). In addition, over this time, a stellar wind becomes established and increases in strength over several days. X-ray spectroscopy of TW Hya and the accompanying spectroscopic diagnostics of density and temperature revealed a large post-shock volume in the corona (Brickhouse et al. 2010). This newly-discovered material has roughly 300 times larger volume and 30 times more mass than the accretion shock itself, and signals the presence of an accretion-fed corona. It appears to be a large turbulent billowing structure in the corona. This process may be similar to the recently identified brightenings 
in the solar corona following the impact of fragments from an erupting solar filament (Reale et al. 2013). Accreting material can supply large nearby magnetic structures and also drive a stellar wind (Cranmer 2009).

While broad ultraviolet, far-UV, and optical emission lines from TW Hya are well documented (Alencar \& Batalha 2002; Herczeg et al. 2002; Dupree et al. 2005a; Ardila et al., 2013) their origin has remained elusive. Intensive study of the X-ray spectrum of TW Hya with CHANDRA coupled with simultaneous optical spectra strongly suggests that the source of its broad optical and X-ray emission arises from the turbulent postshock cooling volume in the stellar atmosphere (Brickhouse et al. 2010; Dupree et al. 2012), and not from 'accretion funnels' approaching the star. The broad emission can suffer some absorption from the accretion stream and can also be substantially modified by a stellar wind which appears to be driven by the accretion process. The structure of this wind, its characteristics as well as the amount of mass loss remain to be determined. These processes have substantial implications for angular momentum loss from the star (Matt \& Pudritz 2005; Matt et al. 2012; Bouvier et al. 2014), for the presence of dust in the surrounding circumstellar material (Alexander et al. 2005), and for an understanding of the accretion process itself and its consequences.

To summarize, the recent measurements suggest a more complicated accretion process than found in the 'standard model' described above. We envision a magnetically channeled accretion flow from the circumstellar disk towards the star. This is defined as an accretion funnel. This flow of plasma accelerates, free-falling towards the star and forms a shock an abrupt increase in plasma temperature and density. The post-shock plasma, a turbulent medium, appears to cool radiatively. The subsequent effects of the accretion shock are many: a turbulent cooling region that produces the broad ultraviolet emission, the broad optical emission lines, and the broad near-IR helium emission; a heated spot or ring in the stellar photosphere that creates the veiling or filling-in of photospheric lines; a large heated volume in the stellar corona detected in O VII; and acceleration of a stellar wind detected as absorption in $\mathrm{H} \alpha$, He I and ultraviolet line profiles.

In this paper, many new sequences of optical, near infrared, and far ultraviolet spectra are presented to infer the characteristics of the accretion process and wind on several time scales. The spectra allow identification of new structures in the stellar atmosphere, give insight into the accretion parameters, and probe the wind from the star. Spectral observations in the far-UV, optical, and near-IR regions are described in Section 2; the source of the $\mathrm{H} \alpha$ line is discussed in Section 3. Spectral indications of the accretion funnels themselves are presented in Section 4. The $\mathrm{H} \delta$ transition is compared to predictions of current MHD models 
in Section 5. The variable warm wind2 extending to greater heights in the chromosphere than H- $\alpha$ is shown by the near-infrared transition of He I (Section 6). Section 7 presents evidence inferred from the far ultraviolet spectra bearing on the variable post-shock conditions. Section 8 evaluates a previous claim of wind presence and characteristics. Discussion and Conclusions occur in Section 9.

\section{Observations}

A variety of spectroscopic observations (Table 1) has been acquired to assess the structure and dynamics of the atmosphere of TW Hya. Optical spectra were obtained during many observing runs at the Magellan-CLAY 6.5m telescope at Las Campanas Observatory, Chile. MIKE, the double-echelle spectrograph (Bernstein et al. 2003) was used with a $0.75^{\prime \prime} \times 5^{\prime \prime}$ slit that yields a 2-pixel resolution element of $\lambda / \Delta \lambda \sim 30,000$ on the red side $(\lambda \lambda 4900-9300)$ which contains the $\mathrm{H} \alpha$ line and $\lambda / \Delta \lambda \sim 37,000$ on the blue side $(\lambda \lambda 3200-5000)$ used for the higher Balmer lines. The IDL pipeline developed by S. Burles, R. Bernstein, and J. X. Prochaska 3 was used to extract the spectra, and IRAF software 4 was employed in the analysis. Additional optical spectra were taken at the FLWO 1.5-m telescope with the Tillinghast Reflector Echelle Spectrograph (TRES). TRES is a temperature-controlled, fiber-fed instrument with a resolving power $\mathrm{R} \sim 44,000$. The spectra were reduced with a custom IDL pipeline 5

Near-infrared spectra were obtained with PHOENIX at Gemini-S during two classical observing runs. The PHOENIX setup consisted of a slit width of 4 pixels yielding a spectral resolution of $\sim 50,000$. The order-sorting filter J9232 was selected which spans $1.077-1.089 \mu \mathrm{m}$ and allows access to the He I line at $1.083 \mu \mathrm{m}$. Standard procedures were followed by acquiring target spectra using a nodding mode $(\mathrm{A}-\mathrm{B})$ with a spatial separation of 5 arcsec. Because standard comparison lamps have a sparse wavelength pattern in this near-infrared region, we observed a bright $\mathrm{K}$ giant containing many securely identified narrow photospheric absorption lines in order to determine the wavelength scale. Other near-infrared spectra were taken with NIRSPEC (McLean et al. 1998, 2000) on the Keck II telescope. The echelle

\footnotetext{
${ }^{2}$ Here we take a warm wind to have a temperature of $\sim 15,000 \mathrm{~K}$ indicative of the formation region of He I; a hot wind is considered to have a temperature of $\sim 10^{5} \mathrm{~K}$ or higher. A stellar wind, if like the solar wind, exhibits a progression in temperature from 'warm' to 'hot'.

${ }^{3}$ See http://web.mit.edu/ burles/www/MIKE/

${ }^{4}$ See iraf.noao.edu

${ }^{5}$ See www.sao.arizona.edu/FLWO/60/tres.html
} 
cross-dispersed mode of NIRSPEC with the NIRSPEC-1 order-sorting filter was selected and the slit of $0.42^{\prime \prime} \times 12^{\prime \prime}$ gives a nominal spectral resolution of 23,600 . The long-wavelength blocking filter was not used in order to minimize unwanted fringing. Internal flat-field lamps, NeArKr arcs, dark frames, and K0 giants were used as calibration exposures. Spectra were taken in a standard nodding mode. Data reduction was carried out with the REDSPEC package specifically written for NIRSPEC (McLean et al. 2003).

Far-UV spectra of TW Hya from FUSE were taken from the MAST archive and the extraction procedure was fully described elsewhere (Dupree et al. 2005a). Some HST spectra of TW Hya obtained with STIS were also taken from the MAST archive at STScI, and from the CoolCAT UV spectral catalogue (Ayres 2005) 6 Other HST spectra came directly from the MAST archive at STScI. Details of these spectra are also given in Table 1.

\section{Identifying the Source of $\mathbf{H} \alpha$}

The $\mathrm{H} \alpha$ profile is useful for probing the accretion process. A broad $\mathrm{H} \alpha$ line has long been considered a signal of ongoing accretion in young stars (Bertout 1989; Hartmann et al. 1994; Alencar \& Batalha 2002), and the cause of the broadening has frequently been ascribed to 'turbulence' (Alencar \& Basri 2000). In this section, we present the most extensive study of TW Hya in the optical region. The $\mathrm{H} \alpha$ profile was measured on 41 nights over a decade (Figure 1), with anywhere from 2 to 300 spectra per night. Most of the observations were taken on sequential days which enables the identification of dynamic events in a stellar atmosphere. As has been noted almost two decades ago (Johns-Krull \& Basri 1997), snapshot spectra do not provide a characteristic representation of a complex accreting system, and time sequences are invaluable. The $\mathrm{H} \alpha$ profiles in TW Hya show dramatic changes in the strength of the emission and the presence of absorption features at negative (outflowing) velocities 7 The signature of a wind in the $\mathrm{H} \alpha$ profile has been noted previously (Hartmann 1982; Calvet \& Hartmann 1992; Gullbring et al. 1996; Alencar \& Basri 2000; Alencar \& Batalha 2002) but only recently has the hourly development of wind absorption became evident (Dupree et al. 2012). The shape of the emission profile indicates material motions of gas at chromospheric temperatures. Comparison of profiles in Figure 1 and evaluation of the flux at negative and positive velocities demonstrates the presence of roughly symmetric $\mathrm{H} \alpha$ profiles (to within $10 \%$ ) centered on TW Hya for $42 \%$ of the spectra and a positive flux enhancement ('red enhanced') for $52 \%$ of the spectra. The full width at half maximum (FWHM) of the lines

\footnotetext{
${ }^{6}$ See casa.colorado.edu/ ayres/CoolCAT/.

${ }^{7}$ Water vapor produces an absorption feature on the positive velocity side near $+50 \mathrm{~km} \mathrm{~s}^{-1}$.
} 
rangew from 200 to $300 \mathrm{~km} \mathrm{~s}^{-1}$. This positional symmetry is consistent with formation on the star itself. When the profiles appear to be 'red-enhanced', this is caused by substantial absorption and scattering (on the "blue-side") at negative velocities by outflowing material forming the stellar wind. A particularly instructive sequence occurred in 2007 Feb (Dupree et al. 2012). At that time, the $\mathrm{H} \alpha$ profile was roughly symmetric on the first night of observation, then the wind opacity systematically increased over the following 3 nights.

These observations demonstrate that the $\mathrm{H} \alpha$ profile from TW Hya is inherently broad and symmetrically positioned on the stellar velocity, but can be substantially absorbed by the stellar wind. In addition, changes in the long-wavelength side of the profile (cf. Figure 1) can result from a combination of enhanced post-shock emission and changing absorption by the accretion stream. The pole of TW Hya is observed directly. It is here that accretion occurs most of the time (Donati et al. 2011), and the shock and post-shock cooling region can be seen. The source of this broad emission has been long debated. With the assumption that emission arises from the accretion stream, models tend to predict pointed profiles from a star observed at low inclination. Frequently absorption on the red side that results from the infalling material emerges from the calculations (Muzerolle et al. 2001). This makes H $\alpha$ appear shifted to shorter wavelengths in contrast to most of these observations. Authors have noted that the magnetospheric accretion models do not adequately represent spectral profiles found in several T Tauri stars (e.g. Alencar \& Basri 2000). Changes in damping parameters and the introduction of Stark broadening, can manage to broaden the $\mathrm{H} \alpha$ profile but the observed profiles in TW Hya have flatter peaks and are frequently wind absorbed in contrast to the calculated profiles (Figure 2).

During many nights the observed $\mathrm{H} \alpha$ profile remains centered on the stellar radial velocity and appears symmetric without a distinct absorption feature. As previously suggested (Dupree et al. 2012), the broad $\mathrm{H} \alpha$ lines can result naturally from turbulent conditions in a post-shock cooling volume. This identification derived from the time study of an X-Ray accretion event which was followed by sequential changes in emission line profiles and fluxes, veiling, and subsequent coronal X-Ray enhancement. The breadth of the $\mathrm{H} \alpha$ line is slightly larger $\left(\sim 200-275 \mathrm{~km} \mathrm{~s}^{-1}\right)$, but comparable to that of Ne IX X-ray lines arising in the shock (126 to $229 \mathrm{~km} \mathrm{~s}^{-1}$ ) while slightly less than the full width of the symmetrized far-ultraviolet lines (C III and O VI) observed to be $\sim 325 \mathrm{~km} \mathrm{~s}^{-1}$ in TW Hya (Dupree et al. 2005a). Moreover, the amount of material in the post-shock region inferred from the X-ray diagnostics (Brickhouse et al. 2010) as well as near-infrared measurements (Vacca \& Sandell 2011) exceeds by a few orders of magnitude the material expected in an accretion column (cf. Dupree et al. 2012). These facts strongly suggest that the symmetric $\mathrm{H} \alpha$ emission line arises predominantly from the turbulent post-shock cooling region. It has been known for a long time that models of a stellar chromosphere alone can not produce strong hydrogen emission 
lines (Calvet et al. 1984). A symmetric $\mathrm{H} \alpha$ profile centered on the stellar radial velocity is expected from TW Hya because the postshock region is in our full view. When a wind from the star is present, the short wavelength side of the profile is weakened, and the resulting profile is stronger on the long wavelength side of the line as frequently appears in TW Hya. Absorption by infalling material can affect the profile as well.

Systems with different inclinations might be expected to produce similar turbulently broadened profiles if the post-shock region is not obscured by a disk. In fact, broad $\mathrm{H} \alpha$ has long been known (Bertout 1989; White \& Basri 2003) to signal the presence of a classical accreting T Tauri star. Differences in the profile may arise from wind scattering which would be determined by the line-of-sight component of the expansion velocity. But as is evident from Figure 1, where the line-of-sight reflects the maximum outflowing velocity from TW Hya, the wind velocity and opacity can change substantially with time. The He I profile, discussed in Section 6 offers an interesting test of the effects of inclination.

\section{Detection of Shadowing by Accretion Funnels}

The $\mathrm{H} \alpha$ profiles also reveal structures that occur in the stellar wind and their behavior becomes apparent in the spectral sequences lasting several days. Alencar and Batalha (2002) remarked on a broad (FWHM $\sim 125 \mathrm{~km} \mathrm{~s}^{-1}$ ) absorption feature in $\mathrm{H} \alpha$ that appeared in nightly spectra suggesting variation in wind opacity. Time domain spectroscopy presented here confirms the changes in the wind, and also reveals another feature. Profiles in Figure 1 show an absorption feature that occurs at $\sim-50$ to $-100 \mathrm{~km} \mathrm{~s}^{-1}$. This feature can change in strength and velocity from night to night, but is constant in velocity over a single night. Particularly instructive are the nights following 2004 Apr 27 (Figure 3) where the absorption feature appears at $-80 \mathrm{~km} \mathrm{~s}^{-1}$, vanishes on Apr 28, re-appears on Apr 29 at $-150 \mathrm{~km} \mathrm{~s}^{-1}$, and on Apr 30 at $-100 \mathrm{~km} \mathrm{~s}^{-1}$. Similar behavior occurred in 2007 Feb and 2009 Jun (Figure 1) and also in 2006 (Figure 4). These absorption features in the wind remain at constant velocity during the night. The three-night sequence from 2006 April is presented in gray scale representation in Figure 5 . The first night shows a narrow $\left(\sim 50 \mathrm{~km} \mathrm{~s}^{-1}\right)$ absorption feature at $-125 \mathrm{~km} \mathrm{~s}^{-1}$ at constant velocity; this feature becomes weaker early in night 2 . However, during the second night of observations, the absorption at $-50 \mathrm{~km} \mathrm{~s}^{-1}$ at the start of the night is replaced by an absorption feature at $-200 \mathrm{~km} \mathrm{~s}^{-1}$ by the end of the night. The right panel of Figure 5 which contains a line plot of the 8 hours of observation during night 2 illustrates this jump quite clearly. In addition, the variable infall of material at high velocities can be seen in the profile. The short rotation period of TW Hya (3.57 days; Huélamo et al. 2008) causes different viewing angles to occur during the 3 successive nights 
of our observations. The narrow absorptions appear constant in velocity suggesting they are located at high latitudes on the star. If the features moved outward within the wind, the speeds of $\sim 150 \mathrm{~km} \mathrm{~s}^{-1}$ would allow material to cover many ( 6) stellar radii over an 8-hour span of observations and this motion would also be visible in the line profiles. But such a shift is not observed.

Such static behavior differs from high-lying stellar prominences found in rapidly rotating stars such as AB Dor (Collier Cameron \& Robinson 1989) and in rapidly rotating T Tauri stars (Oliveira et al. 2000; Skelly et al. 2008; Günther et al. 2013) where the absorption feature moves rapidly in velocity from negative to positive (or vice versa) as the feature traverses the stellar disk. The absorption features in TW Hya are stable in velocity for hours, and are not at all similar to the moving Discrete Absorption Components (DACs) observed in the winds of O stars (cf. Howarth et al. 1995) which generally are very broad $\left(\sim 400 \mathrm{~km} \mathrm{~s}^{-1}\right)$ and move at thousands of $\mathrm{km} \mathrm{s}^{-1}$ through the atmosphere (cf. Kaper et al. 1999); only absorption features in one Wolf-Rayet star (HD 50896) are broad, but static (Massa et al. 1995).

The static nature of these relatively narrow absorptions in TW Hya suggests they arise in a stable cool structure located in the wind itself that is silhouetted in the hydrogen line profile. These could be the signature of the accreting columns crossing the path of the wind. In an accelerating wind, features located at -50 to $-150 \mathrm{~km} \mathrm{~s}^{-1}$, would exist at about $1.2-1.3 \mathrm{R}_{\star}$ (Dupree et al. 2008), judging from the chromospheric helium line asymmetries, and above the shocked material produced by accretion (Sacco et al. 2010). The strength of the absorptions, from 0.4 to 0.6 of the local continuum provided by the $\mathrm{H} \alpha$ emission, indicates an optical depth, $\tau$ of $\sim 0.7$. Here we take $\tau=\kappa \rho H$ where $\kappa\left(T, n_{2} / n_{1}\right)$ is the line absorption coefficient (incorporating a dependence on temperature and the level population ratio of hydrogen), $\rho$ is the hydrogen density, and $H$, the thickness of the line-forming region. The temperature and density structure of the 'funnel flows' is uncertain. However, the preshock electron density was determined (Brickhouse et al. 2010) to be $5.8 \times 10^{11} \mathrm{~cm}^{-3}$ which implies a hydrogen density of $5 \times 10^{11} \mathrm{~cm}^{-3}$ for a plasma with $10 \%$ helium. For a temperature $\mathrm{T} \sim 7000 \mathrm{~K}$, the observed broadening and our non-LTE calculations 8 for chromospheric hydrogen suggest that the $\mathrm{n}_{2} / \mathrm{n}_{1}$ level populations of the $\mathrm{H} \alpha$ line range from $6.4 \times 10^{-7}$ to $1.8 \times 10^{-8}$ in this temperature and density regime (Dupree et al. 2008). The scale length to produce the narrow absorption is small, less than $0.01 \mathrm{R}_{\star}$. Another model of the accreting stream (Hartmann et al. 1994) gives $\mathrm{T}=7000 \mathrm{~K}$ and the level 2 population of hydrogen as $8 \times 10^{5} \mathrm{~cm}^{-3}$ near the

\footnotetext{
${ }^{8}$ The PANDORA code (Avrett \& Loeser 2008) was used in a spherical expanding semi-empirical model constructed to match wind scattering profiles of $\mathrm{H} \alpha$, He I, and the chromospheric density inferred from the ultraviolet C II lines.
} 
star, such that the inferred thickness is much smaller, $\sim 100 \mathrm{~km}$. In either case, formation in a narrow accretion funnel appears plausible to account for these absorption features.

Chromospheres, winds, and wind structures produce asymmetric lines and absorption features that may be broad (such as a central reversal) or narrow as discussed here. The uniqueness of these narrow 'notches' relates to their constant velocity in the $\mathrm{H} \alpha$ line profile. A blocking structure located in the wind could be seen at all angles where it is observed against a region producing emission. Many studies of emission from T Tauri stars have been published but very few have the time sampling of multiple sequential spectra that are presented here.

\section{The $\mathbf{H} \delta$ Line}

Sophisticated models of magnetospheric accretion processes have also been constructed and used to predict hydrogen line profiles. A sequence of Balmer lines calculated by Kurosawa \& Romanova (2013) suggested that the $\mathrm{H} \delta$ transition at $4101 \AA$ is an optimum line to reveal persistent red-shifted sub-continuum absorption resulting from the inflowing accretion stream in the 'standard' model. Absorption is expected when the hotspot is in the line of sight of the observer as is the accretion stream. TW Hya is a good test of these assumptions. Because of its pole-on orientation, the accretion stream and hot spot are in the line of sight. Our MIKE spectra capture the pivotal $\mathrm{H} \delta$ line, as well as the complete Balmer series. Figure 6 shows four lines of the Balmer series $(\mathrm{H} \alpha, \mathrm{H} \beta, \mathrm{H} \gamma, \mathrm{H} \delta)$ taken over 4 sequential nights in 2007 (Dupree et al. 2012). During this period the $\mathrm{H} \alpha$ line displays a slight inflow asymmetry (blue side stronger than red side) early on the first night, followed by the subsequent onset of a wind. $\mathrm{H} \beta$ and $\mathrm{H} \gamma$ exhibit an absorption feature at $\sim+50 \mathrm{~km} \mathrm{~s}^{-1}$ which signals enhanced inflow following the X-ray accretion event (Dupree et al. 2012). However the predicted sub-continuum absorption signalling infalling material is not evident in the $\mathrm{H} \delta$ line. The period of radial velocity variation, namely 3.57 days (Huélamo et al. 2008) could cause a change in the absorption strength if there were substantial modulation in the orientation of the hot spot and the accretion column. The lack of an absorption feature in the $\mathrm{H} \delta$ profile suggests that the $\mathrm{H} \delta$ emission does not arise from infalling material, but must originate from another region. Another sequence of $\mathrm{H} \delta$ profiles over 4 consecutive nights is shown in Figure 7. The first night of the sequence shows enhanced emission with shallow broad absorption between +100 to $+200 \mathrm{~km} \mathrm{~s}^{-1}$, inferred from the line asymmetry. This pattern may be the signal of an accretion event. However, subsequent nights (July 14-16) reveal a symmetric profile, indicating the infall has lessened, and the symmetry and FHWM $\left(\sim 150 \mathrm{~km} \mathrm{~s}^{-1}\right)$ are consistent with formation in a turbulent post-shock cooling volume. Variability of the 
accretion rate is also documented by the X-ray diagnostics (Brickhouse et al. 2012) which have shown a five-fold reduction in the rate from exposures separated by 2.7 days. Other studies have measured $\mathrm{H} \delta$ in T Tauri stars (Edwards et al. 1994; Petrov et al. 1996; Alencar \& Basri 2000) and the profiles in the majority of stars do not resemble the models presented in Kurosawa \& Romanova (2013).

\section{Variable Warm Wind in Near IR Helium}

The broad near-IR line of He I at $10830 \AA$, because of its width (FWHM $\sim 200 \mathrm{~km} \mathrm{~s}^{-1}$ ), also appears formed in the post-shock material. This feature has become a useful probe of the wind and infall environment of accreting T Tauri stars (Dupree 2003; Edwards et al. 2003; Dupree et al. 2005a; Edwards et al. 2006). For TW Hya, we have acquired near-IR spectra spanning 8 years including sequences of consecutive nights. A comparison of the spectra obtained during this 8 year span is shown in Figure 8. Over this long time scale, practically every characteristic of the P Cygni profiles changes: the emission level, the wind speed and opacity, and the appearance (or not) of inflowing material. The emission strength varies by more than a factor of 2 ; the terminal velocity of the wind varies from -200 to -315 $\mathrm{km} \mathrm{s}^{-1}$; the wind opacity changes over the absorption profile; the infall terminal velocity varies from +270 to $+363 \mathrm{~km} \mathrm{~s}^{-1}$ as does the opacity of the infalling material. Speaking precisely, an outflow of material is observed in the near-infrared helium profile of TW Hya. The escape velocity from the photosphere is $530 \mathrm{~km} \mathrm{~s}^{-1}$ for TW Hya assuming $\mathrm{M}=0.8 \mathrm{M}_{\odot}$ and $\mathrm{R}=1.1 \mathrm{R}_{\odot}$ (Donati et al. 2011). At a distance of 1 stellar radius above the surface, this value decreases to $400 \mathrm{~km} \mathrm{~s}^{-1}$, which is comparable to the observed typical terminal velocities. It is highly likely then that the outflows observed in the near-IR helium line and the sequential acceleration with temperature displayed by the UV lines in Dupree et al. (2005a) form a true stellar wind 9

The time-domain spectroscopy revealing these changes suggests that all T Tauri objects undoubtedly exhibit similar variability that snapshot studies (Edwards et al. 2006) can not discern. The extent of the variation also blurs the search for correlations of wind parameters with geometric inclination of the star or disk (Appenzeller \& Bertout 2013).

The helium absorption profile is broad, signalling its origin in a stellar wind, through its classic P Cygni shape. There is no sign of a narrow low-velocity ( 10 to $\left.100 \mathrm{~km} \mathrm{~s}^{-1}\right)$ feature such as identified in several spectra of other T Tauri stars (Edwards et al. 2006) and

\footnotetext{
${ }^{9}$ Other studies (Batalha et al. 2002 ) suggest $\mathrm{M}=0.7 \mathrm{M}_{\odot}$ and $\mathrm{R}=0.8 \mathrm{R}_{\odot}$ which would indicate a comparable escape velocity $\left(410 \mathrm{~km} \mathrm{~s}^{-1}\right)$ at 1 stellar radius above the photosphere.
} 
conjectured to originate from the circumstellar disk. In the case of TW Hya, $\mathrm{H}_{2}$ and $[\mathrm{O} \mathrm{I}]$ emission arise from the disk (Herczeg et al. 2002, Pascucci et al. 2011) since their velocities coincide with that of the star itself. The infrared [Ne II] transition exhibits a low velocity $(-5$ $\mathrm{km} \mathrm{s}^{-1}$ ) outflow which suggests the presence of a cool $(1000 \mathrm{~K})$ photoevaporative molecular wind (Rigliaco et al. 2013). It is hard to see how helium which is more highly excited would be produced in such a wind.

Several sequences of the He I line over consecutive nights are shown in Figure 9. At these times minimal variation of the line profiles occurred on a short time scale: a small change in the emission in 2009; changes in the inflow opacity in 2010. During 2009 and 2010 the wind absorption had constant high optical depth (a flat-bottomed profile) over a 50 to $100 \mathrm{~km} \mathrm{~s}^{-1}$ span, and subtle changes in opacity would not be observable. In contrast, the $\mathrm{H} \alpha$ profile over sequential nights reveals changes in wind opacity and absorption troughs (cf. Fig. 1 A-E, I, K, L). In 2009, the absorption feature at $-50 \mathrm{~km} \mathrm{~s}^{-1}$ is also found in the $\mathrm{H} \alpha$ profile from those nights (see Figure 1). At that time, the $\mathrm{H} \alpha$ line revealed wind scattering that extends to $-100 \mathrm{~km} \mathrm{~s}^{-1}$, and the helium wind extended to $-200 \mathrm{~km} \mathrm{~s}^{-1}$, an indication of accelerated expansion.

The emission strength exhibits substantial change. Of course, the amount of emission depends on the intrinsic line strength which is then modified by the wind scattering and some absorption from infalling material. Close inspection of the profiles suggests that the emission itself is indeed changing. On one occasion, 2002 May 20, the profile displays emission above the continuum level extending to both -300 and $+300 \mathrm{~km} \mathrm{~s}^{-1}$ indicating a strong and broad intrinsic emission component.

The inflow signature in helium is a particularly useful diagnostic of the accretion process. In a standard magnetospheric accretion model (Brickhouse et al. 2012), the terminal velocity of the infalling material, determined by the free-fall distance from the circumstellar disk, controls the temperature of the shock. X-ray spectra indicate that the shock temperature, measured directly from the ratio of forbidden plus intercombination to resonance line fluxes of Ne IX, varied by a factor of 1.6 over a span of 13 days between CHANDRA pointings (Brickhouse et al. 2012). The shock temperatures vary between 1.9MK and 3.1MK. In this standard model, the post-shock temperature is given by $T_{p o s t} \sim 3 m v_{f f}^{2} / 16 k$, where $v_{f f}$ is the free-fall velocity, $k$ is the Boltzmann constant and $m$ is the mean atomic mass (Brickhouse et al. 2010). The measured shock temperatures from 3 CHANDRA pointings in 2007 suggest that terminal free-fall velocities from 380 to $485 \mathrm{~km} \mathrm{~s}^{-1}$ occurred.

In the model (Brickhouse et al. 2012), when the free-fall velocity is low, the disk supplying the accreting material originates closer in to the star. The filling factor on the stellar surface increases which implies an increase in the mass accretion rate, and also in the mate- 
rial in the post-shock cooling zone. A range of parameters for this model is shown in Figure 10 (left panel). This scenario is confirmed by the behavior of the helium line. The amount of emission in the He I line appears inversely linked to the terminal velocity of the inflowing material (Figure 10, right panel). Lower values of the inflow terminal velocity lead to much stronger emission arising in the post-shock region, which is consistent with the higher mass accretion rate as predicted in the dipole model. The helium absorption indicates lower velocities than the terminal velocities of the models suggesting that the absorption arises from a volume above the accretion shock. It is also quite likely that the neutral helium is ionized by the X-ray emission from the shock as the helium approaches in the pre-shock stream (Lamzin 1999; Gregory et al. 2007; Brickhouse et al. 2010), and as a result does not reach a terminal free-fall velocity.

It is interesting that the helium line gives evidence of subcontinuum absorption due to inflow, when the hydrogen lines generally do not show such strong signatures of inflowing material. On 2007 March 1, we obtained (almost) simultaneous spectra of helium (Figure 8 ) and the hydrogen lines (shown as night 4 in Figure 6). The reversal of the negative velocity side of the Balmer lines on to the positive velocity side (Figure 6) indicates weak broad absorption near $+200 \mathrm{~km} \mathrm{~s}^{-1}$ on the $\mathrm{H} \beta, \mathrm{H} \gamma$, and $\mathrm{H} \delta$ transitions. However the helium line displays substantial absorption extending to $+325 \mathrm{~km} \mathrm{~s}^{-1}$. The temperature structure along the accretion column is not known but it appears likely that helium forms at higher temperatures where the hydrogen is ionized, and these temperatures are associated with higher infalling velocities. This could create a higher opacity in helium with the ability to probe a different, potentially more extended accreting region than represented by the hydrogen lines.

Because stronger emission signals enhanced accretion of material, this material might cause an increase in both the wind opacity and wind speed if the accretion contributes to wind acceleration. There is a hint of this in nine out of the ten near-IR spectra of He I (Figure 11). The one outlier with the fastest outflow speed belongs to the 2007 March 1 observation discussed above. Not only does this profile exhibit the highest outflow velocity, but also the largest subcontinuum 'blue absorption' of all ten nights of observations. It is difficult to quantify this further because the intrinsic profile of the helium line is unknown. The profile exhibits absorption on the 'red' side caused by accretion, and substantial scattering on the 'blue' side in the stellar wind. Figure 11 shows that increased emission in He I $10830 \AA$ may be linked directly to a faster outflowing terminal velocity, giving evidence for an accretion-driven stellar wind. 


\section{Variable Post-Shock Conditions from UV lines}

The UV and far-UV lines also exhibit changes in flux as evidenced by HST and FUSE spectra. The expanding atmosphere of TW Hya is revealed by the asymmetric profiles of the major resonance lines (Dupree et al. 2005a), but substantial changes in the profiles are evident at different times. Figure 12 (left panel) contains resonance line profiles of C IV

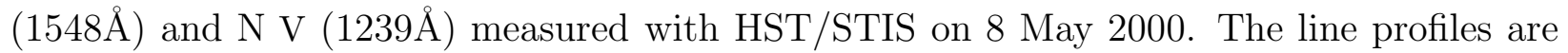
strikingly similar to one another, confirming formation in the same region of the atmosphere. Ardila et al. (2013) also remarked recently on this C IV/N V similarity from a much larger sample of accreting stars. The temperatures of formation of $\mathrm{C} \mathrm{IV}$ and $\mathrm{N} \mathrm{V}$ in a collisionally ionized plasma are similar at $1-2 \times 10^{5} \mathrm{~K}$. The profiles exhibit both extended emission to positive velocities, and the characteristic sharp cutoff caused by wind absorption at negative velocities. More wind opacity is evident in the $\mathrm{C}$ IV profile than in $\mathrm{N}$ V. This is to be expected since the strength of absorption is proportional to the value of $g f \lambda \times N_{i} / N_{H}$ where $N_{i}$ is taken to be $N_{C}, N_{O}$, or $N_{N}$ giving the appropriate elemental abundance. The value of $g f \lambda \times N_{C} / N_{H}$ for carbon is larger than the similar quantity for the $\mathrm{N} \mathrm{V}$ transition. Note the extended wing at positive velocities on this date giving a half-maximum value of +200 $\mathrm{km} \mathrm{s}^{-1}$. Contrast these profiles with the resonance lines of $\mathrm{O}$ VI and C III on 20 Feb 2003 (Figure 12, right panel) measured with FUSE. Both of these lines are also similar to one another, and their formation temperatures are typically $8 \times 10^{4}-3 \times 10^{5} \mathrm{~K}$ in equilibrium conditions. The $\mathrm{C}$ III profile exhibits more wind opacity than O VI which again can be understood from the difference in the quantity $g f \lambda \times N_{C} / N_{H}$ which is larger for C III than for $\mathrm{O}$ VI by a factor of 2.8. The relative amount of absorption in the wind is in harmony with atomic physics. Thus, based on these spectra, we conclude that the UV and far-UV lines are produced in the same plasma region, but conditions in this region change dramatically from time to time.

Intercomparison of two of the ultraviolet profiles (Figure 13) reveals the meaningful differences. Excess emission on the positive velocity side of the profile in 2000 does not occur in the 2003 observation. The half-maximum value of the $\mathrm{O}$ VI profile in 2003 extends only to $+125 \mathrm{~km} \mathrm{~s}^{-1}$, whereas C IV extended to $+200 \mathrm{~km} \mathrm{~s}^{-1}$ in 2000 . Additionally there is greater wind scattering on the negative velocity side of the profile in C IV as compared to O VI. While changes in the wind opacity between the two observations are unknown, the atomic physics of these lines must be a first consideration. C IV possesses a $17 \%$ larger atomic parameter $\left(g f \lambda \times N_{i} / N_{H}\right)$ than O VI (where $N_{i}=N_{C}$ or $N_{O}$ ) which contributes to its greater opacity. We also do not know the distribution of material with temperature in the wind which can affect both the ionization equilibrium and the formation of the lines.

Variation of the C II and C IV emission on shorter time scales can be found in Figure 14 
from HST/STIS spectra. Substantial changes are apparent on a time scale of years between the HST measurements, and also over a time scale of 6 days which separates the 2010 Jan 29 and 2010 Feb 4 observations of C IV. The C IV emission appears to strengthen with C II, however the 4 simultaneous measurements show scatter. The C II line is not broadened as much as the C IV emission, suggesting that the contribution from the turbulent post-shock cooling region is less. The positive velocity side of the C II emission ( $\lambda 1335.7)$ resembles the positive velocity side of the $\mathrm{H} \delta$ line (cf. Figure 7 ) indicating that both may be formed in a region distinct from the higher temperature species (C III, C IV, etc.).

A closer look at the variations in the high temperature emissions is provided by the sequence of individual segments of the FUSE O VI spectra. Under collisionally-dominated equilibrium conditions, $\mathrm{O}$ VI is formed at a temperature of $3 \times 10^{5} \mathrm{~K}$. However, the $\mathrm{O}$ VI line continues to be formed in hotter coronal plasmas due to the extended wing of Lilike ions in the ionization balance at high temperatures. O VI has been used consistently as a diagnostic of the dynamical properties of outflows in the solar corona (Kohl et al. 2006) and X-ray emitting shocks in OB-star winds (Lehner et al. 2003). Evidence of the dramatic changes in emission from TW Hya can be found from the individual C III and O VI profiles assembled over a 32 hour span and shown in Figure 15. Each segment represents an integration time ranging from 2600 to $3700 \mathrm{~s}$. The impression that C III varies more than $\mathrm{O}$ VI is quantitatively confirmed by an extraction of the line fluxes. C III varies by a factor of $\sim 1.8$ and $\mathrm{O}$ VI by a factor of $\sim 1.5$. Evaluating the negative and positive velocity sides of each line shows that the fluxes are not correlated between the sides. However the uncertainty in flux on the negative velocity side is larger because the total counts are lower. And the total counts in the O VI line are a factor of 4 or more than in the C III line, making the O VI parameters a more secure measurement. The relatively constant profile of the outflowing wind absorption at negative velocities contrasts dramatically with the changing emission at positive velocities during this time. Such profile variation provides additional evidence that the emission arises from the turbulent post-shock accretion volume, and the the line is scattered in the hot wind which is present during these observations. Similar behavior in He I 5876 ̊ was found in another accreting T Tauri star, RU Lupi (Gahm et al. 2013).

Fluxes taken from the individual spectra are shown in Figure 16 where in the first pointing the C III ( $\lambda$ 977) line is stronger than O VI $(\lambda 1031.91)$; this behavior is reversed in the second pointing about one day later. Here we have selected segments where the $\mathrm{SiC}$ detector (containing the C III line) is well-aligned with the LiF detector (containing the O VI line) 10 he FUSE telescope contained 4 coaligned optical channels. Pointing on a target

${ }^{10} \mathrm{~T}$ 
was maintained by the fine error sensor which viewed one of the channels. Because thermal changes on orbit caused rotation of the mirrors, this could lead to misalignment of the optical channels and a target could drift in and out of the aperture in the channels not used for guiding. This could affect the measurement of the C III line since it occurs in a channel not used for guiding. In our analysis, segments for C III were selected when the channels were coaligned. However, the C III atom has a metastable level whose population is dependent on density which could contribute to the behavior of C III. And of course, the post-shock cooling region can change as well. During the second pointing when both lines could be measured simultaneously over $\sim 5$ hours, the fluxes are correlated with a linear correlation coefficient of 0.9 .

\section{Remarks concerning a Hot Wind}

The presence of a hot wind from TW Hya has been questioned by Johns-Krull and Herczeg (2007), hereafter JKH. These authors offer several arguments purporting to show that the wind is not hot. We argue below why we believe that their concerns are not valid, and discuss four relevant issues.

(1) As evidence for outflow, JKH require sub-continuum absorption in a line profile to be present at 'blue-shifted' wavelengths. While that phenomenon is well-understood from characteristic P Cygni line profiles, the signature of outflowing material can be revealed in other ways. A shift of the apparent wavelength of the centroid of an emission or absorption line can reveal mass motions. Mass motions can also be signaled by line asymmetries. Hummer and Rybicki (1968) first pointed out that apparently redshifted line profiles can arise in a differentially expanding atmosphere; in fact these have been widely observed in luminous stars (cf. Mallik 1986, Robinson \& Carpenter 1995, Dupree et al. 2005b, Lobel \& Dupree 2001) and in the Sun (Rutten \& Uitenbroek 1991). The assertion by JKH that sub-continuum absorption must be present to identify outflow neglects both line-transfer physics and stellar observations themselves.

(2) In an attempt to demonstrate the absence of absorption in resonance doublets, JKH arbitrarily scale and overlay the long wavelength member of the C IV doublet on to the short wavelength member of the doublet by forcing both the line peaks and blue wings to match. They claim that signs of absorption are missing in the red wing of the $1548 \AA$ line that would be caused by the wind absorption in the $1550 \AA$ line. Such arbitrary scaling is not justified. Atomic physics, supported by laboratory measurements, specifies the wavelength separation between these lines as well as the ratio of the line oscillator strengths. In an effectively optically thin doublet such as C IV, the line flux ratio $(\lambda 1548 / \lambda 1550)$ is 2 . Since 
it is preferable not to amplify the errors in the weaker line of the doublet, here we place the stronger $(1548 \AA)$ line onto the weaker $(1550 \AA)$ transition. The original HST data used by JKH, namely O58D0130, has been superseded by a more optimal spectrum reduction, namely CoolCAT (Ayres 2010) and we also consider that reduction (see Table 1). In Figure 17, we show the CoolCAT reprocessed profile as well as the original spectrum used by JKH.11

When the C IV $1548 \AA$ line is properly overlaid on the $1550 \AA$ line by shifting it according to the wavelength separation $(2.577 \AA$, Griesmann \& Kling 2000), and scaling the flux of $1548 \AA$ by 2 , two facts are immediately apparent from either data reduction: (1) the wind opacity in the resonance line $1548 \AA$, indicated by the negative velocity side of the profile, is greater than in the subordinate line $1550 \AA$, as expected from atomic physics, and (2) excess wind absorption does indeed occur in the long wavelength wing of $1548 \AA$ caused by wind absorption from the $1550 \AA$ line. Consideration of the errors in the measured fluxes at individual wavelengths in the HST/STIS spectrum indicates that the separation visible in Figure 17 between the $1550 \AA$ line and the shifted scaled $1548 \AA$ line ranges from $\sim 2.5$ to $3.2 \sigma$ in the velocity region from +175 to $+350 \mathrm{~km} \mathrm{~s}^{-1}$. Moreover, in this region the dashed line in Figure 17 systematically lies below the solid curve. Thus the effects of hot wind absorption are clearly evident in the C IV profiles.

(3) Trying to demonstrate the absence of wind absorption by the O VI 1037.61 $\mathrm{A}$ transi-

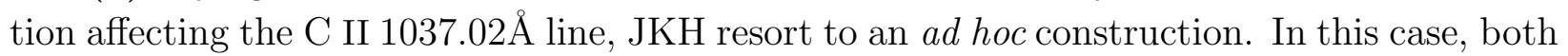
the activity of TW Hya and atomic physics undermine their arguments. The O VI 1037.61 line, has a neighboring $\mathrm{C}$ II line separated by $-172 \mathrm{~km} \mathrm{~s}^{-1}$ which places the $\mathrm{C}$ II line in the velocity range of scattering produced by a hot $\mathrm{O}$ VI wind. To attempt to show that the $\mathrm{C}$ II line is not weakened by scattering, JKH predict the intrinsic strength of a blended O VI and C II feature. By taking the C II line profile at $1335.71 \AA$ observed in 2000 with HST/STIS, these authors scale the profile to mimic the $\mathrm{C}$ II transition at $1037.02 \AA$, and add it to the line profile of O VI $1031.91 \AA$ observed in 2003. The resulting scaled combination is then

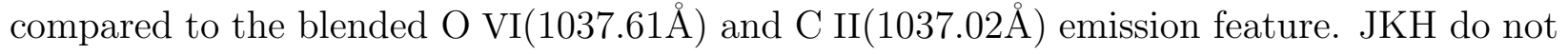
present the parameters used for this scaling, shifting, and combination.

From first principles, the intrinsic relative strength of the C II $1335.7 \AA$ line to the C II $1037.02 \AA$ line is hard to predict. The ratio of $g f \lambda$ values gives a factor of 5.3 for the $\lambda \lambda 1335.7 / 1037.02$ ratio, and the Boltzmann factor ratio could be 2.4 at a temperature of $40000 \mathrm{~K}$ suggesting the $1037.02 \AA$ line could be 13 times weaker than the $1335.71 \AA$ line under optically thin conditions in LTE, and not 1.5 times weaker as JKH propose. However

\footnotetext{
${ }^{11}$ More recent papers (Yang et al. 2012) than Herczeg et al. (2002) estimating the UV line fluxes from TW Hya using revised calibrations report a substantially increased value of the C IV flux by a factor of $\sim 5$.
} 
unknown optical depths and non-LTE conditions occur in the formation of resonance lines of C II causing them to appear weaker and decrease the $\lambda \lambda 1335.7 / 1037.02$ ratio. These issues confound any meaningful scaling. Moreover, the line profiles themselves change with time as demonstrated earlier. Comparison of HST spectra from 2000 to FUSE spectra from 2003 (cf. Figure 13) clearly shows the substantial changes in the emission profiles of ultraviolet lines on the 'red' sides $\left(+100\right.$ to $\left.+400 \mathrm{~km} \mathrm{~s}^{-1}\right)$ between these three years. It is obvious from HST spectra of C II in 2000, 2002, and 2010 (Figure 18) that the critical region of the $1335.7 \AA$ line profile used to assess absorption, namely the region from +35 to $+85 \mathrm{~km} \mathrm{~s}^{-1}$, varies in both absolute flux as well as slope. The claim made by JKH - that this scaled, shifted, blended feature does not exhibit strong absorption on the red side caused by the hot O VI wind - is unjustified, in particular given the substantial changes in both the emission and the wind.

(4) JKH suggest that two $\mathrm{H}_{2}$ lines arising from the circumstellar disk exhibit behavior that suggests a cool wind is present, but not a hot wind. Their interpretation does not agree with the geometry of TW Hya and its disk, nor with the known characteristics of a wind from a dwarf star.

The $\mathrm{H}_{2}$ line at $1333.85 \AA$ occurs $-165 \mathrm{~km} \mathrm{~s}^{-1}$ from the $\mathrm{C}$ II line at $1334.5 \AA$ (at -430 $\mathrm{km} \mathrm{s}^{-1}$ in Figure 18). JKH claim that this $\mathrm{H}_{2}$ feature is absorbed by the $\mathrm{C}$ II wind because their model overpredicts its strength in their spectrum taken in 2000. By contrast, an $\mathrm{H}_{2}$ line lying at a velocity of $-165 \mathrm{~km} \mathrm{~s}^{-1}$ from the C IV $1548 \AA$ feature does not suffer wind absorption when compared to their model (Herczeg et al. 2002). Thus JKH conclude that a cool wind exists, but not a hot wind.

Many spectra subsequent to the one used by JKH reveal conflicting patterns (see Fig. 18). In 2002, the $\mathrm{C}$ II wind extends to $-251 \mathrm{~km} \mathrm{~s}^{-1}$, which would weaken not only the line located at $1333.85 \AA$, but should weaken another $\mathrm{H}_{2}$ line located at $-225 \mathrm{~km} \mathrm{~s}^{-1}$ (corresponding to $-500 \mathrm{~km} \mathrm{~s}^{-1}$ in Figure 17), but it does not. Additionally, in the presence of a strong C II wind, another $\mathrm{H}_{2}$ line emerges at $1335.2 \AA\left(\sim-125 \mathrm{~km} \mathrm{~s}^{-1}\right.$ in Fig. 18; the $\mathrm{R}(2)$ 0-4 transition) which did not appear previously. All of these spectra indicate optically thick winds in C II are present and the wind opacity is variable. Furthermore, the $\mathrm{H}_{2}$ lines behave in apparently unpredictable fashion - perhaps because they are produced through photoexcitation by variable Lyman- $\alpha$ emission. With a more precise location of the $\mathrm{H}_{2}$ emission, the behavior of the molecular lines might aid in the wind description. However, since these lines are pumped by H-Ly $\alpha$, which itself varies, trying to pin down a consistent model may prove challenging. Moreover, serious considerations discussed earlier in (3), rule out the putative effects of wind absorption.

Trying to assess potential effects of a wind upon the observed $\mathrm{H}_{2}$ emission involves many 
factors. First, the line opacity in the wind must coincide in wavelength with the $\mathrm{H}_{2}$ emission from the disk. $\mathrm{H}_{2}$ is believed to be produced less than $2 \mathrm{AU}$ from the star (Herczeg et al. 2002) where it originates in the gaseous component of the circumstellar disk around TW Hya. Recent ALMA observations suggest the dust-depleted cavity lies within 4 AU of the star (Rosenfeld et al. 2012). Ions in a radially-moving stellar wind passing over a distant disk will absorb at lower velocities than when observed against the nearly pole-on star itself. Rosenfeld et al. (2012) suggest the TW Hya disk might have a warp of $\sim 8$ degrees, such that the effective velocity of any absorption would occur at a still lower velocity. Thus the wind may not have significant opacity at the position of the $\mathrm{H}_{2}$ emission when passing across the disk at a low angle. Moreover, any channeling, flux-tube expansion, or inhomogeneities (all of which occur in the solar wind) will modify the amount of absorption and the velocity at which it occurs. A more fundamental concern appears to be the ionization stage in the wind itself. If the stellar wind at $2 \mathrm{AU}$ does not contain $\mathrm{C}$ II or $\mathrm{C}$ IV, then absorption will not occur. From very detailed knowledge about the wind of one star, our Sun, the low species ions are effectively absent at a distance of 1 AU (Landi et al. 2012) and the wind contains the ionization stages of the corona. Thus at a distance of 1 AU or larger, the wind of TW Hya, if similar to the solar wind, would appear to lack the ions that JKH invoke for their arguments.

The presence of a hot wind from TW Hya is not unexpected. The Sun is a low mass star, with weak magnetic fields and it possesses an outer atmosphere with temperatures of a few MK. Wave-heating mechanisms (Kohl et al. 2006) or possibly nano-flares resulting from reconnection (Tripathi et al. 2010) can heat the corona. Self-consistent models for hot winds have been constructed for accreting T Tauri stars (Cranmer 2008). These winds are turbulence driven, and the turbulence has two sources: interior convection and 'ripples' from nearby accretion shocks. Both observations and theory offer much evidence that low-mass cool stars can support a hot outer atmosphere.

We conclude that the stellar wind of TW Hya is a hot wind, evidenced by the ultraviolet spectra as originally suggested (Dupree et al. 2005a).

\section{Discussion and Conclusions}

The many spectra of TW Hya reported here document the source and components of emission line features and the variability of the accretion process, subsequent atmospheric heating, and wind expansion. We find:

1. Time-domain spectroscopy reveals symmetric $\mathrm{H} \alpha$ profiles in approximately half of 
our spectra and indications of outflowing wind asymmetries in the remaining spectra. This spectroscopic example indicates that the source of the broad emission feature arises from the TW Hya star itself, and can be identified with the post-shock cooling zone. Broad lines ranging from X-ray through infrared transitions can be straightforwardly explained by the presence of a variable turbulent post-shock cooling volume producing the emission which can be modified by wind scattering and/or material infall. Earlier evidence of a logical progression in line shape and flux following an X-Ray accretion event also supports this interpretation (Dupree et al. 2012). Models are now needed for the temperature structure and emergent emission from this post-shock region. A recent MHD simulation (Matsakos et al. 2013) of a two-dimensional accretion shock impacting a chromosphere suggests that substantial chaotic motions can result in the low chromosphere. These need to be followed up with calculation of the radiation emitted from the cooling plasma.

2. Absorption features at negative velocities in the $\mathrm{H} \alpha$ line noted by Alencar et al (2002), are demonstrated with the frequent continuous spectral coverage reported in this paper, to be stable in velocity during a night's observation. Moreover, their stable behavior and opacity characteristics suggest they appear to arise from the silhouette of an accretion stream high in the stellar chromosphere. At positive velocities, absorption in $\mathrm{H} \alpha$ mimics the infall speeds detected in He I.

3. Current models (Muzerolle et al. 2001; Kurosawa \& Romanova 2012, 2013) that consider the optical emission from accretion 'funnels' do not agree with the observed line profiles in TW Hya. The observed profiles lack the shift, the asymmetries and/or absorption predicted from accretion stream models. In addition, many models do not include a stellar wind, which is clearly indicated in the observed line profiles, nor emission from a post-shock cooling region.

4. A hot wind, and its variable speed and opacity are revealed by the UV and far-UV resonance lines with spectra spanning a decade in time. Previous arguments offered (JKH) against the presence of a hot wind are shown to be unpersuasive. Broad line profiles and variability support both formation in a turbulent medium and the presence of a hot wind. This hot wind is a natural extension of the wind indicated by the near-IR helium line profile.

5. The He I near-IR transition at $10830 \AA$ shows less night-to-night absorption variability than $\mathrm{H} \alpha$, suggesting a large-scale wind outflow from the star. The emission in the helium line correlates inversely with the velocity of the accreting inflowing material consistent with a simple model of dipole accretion, and formation of the emission in the turbulent post-shock cooling zone. The stellar wind velocity appears related to the strength of helium emission providing direct spectroscopic evidence that the accretion process leads to wind acceleration. 
We thank the anonymous referee for his/her comments that improved the manuscript. The authors gratefully acknowledge the helpful support from Gemini-S astronomers, the staff at Magellan, and KECK II while acquiring these spectra. Observers at FLWO were a great help on short notice. This research has made use of NASA's Astrophysics Data System Bibliographic Services. Some of the data presented here was obtained from the Mikulski Archive for Space Telescopes (MAST). STScI is operated by the Association of Universities for Research in Astronomy, Inc. under NASA contract NAS5-26555. Support for MAST for non-HST data is provided by the NASA Office of Space Science via grant NNX09AF08G and by other grants and contracts. We wish to extend special thanks to those of Hawaiian ancestry from whose sacred mountain of Mauna Kea we are privileged to conduct observations. Without their generous hospitality, the Keck results presented in this paper would not have been possible.

Facilities: Gemini:South (PHOENIX), Magellan:Clay (MIKE), HST(STIS), FUSE, FLWO:1.5m (TRES), Keck II (NIRSPEC)

\section{REFERENCES}

Alencar, S. H. P., \& Basri, G. 2000, AJ, 119, 1881

Alencar, S. H. P., Basri, G., Hartmann, L, \& Calvet, N. 2005, A\&A, 440, 595

Alencar, S. H. P., \& Batalha, C. 2002, ApJ, 571, 378

Alencar, S. H. P., Johns-Krull, C. M., \& Basri, G. 2001, ApJ, 122, 3335

Alexander, R., D., Clarke, C. J., \& Pringle, J. E. 2005, MNRAS, 358, 283

Andrews, S. M., Wilner, D. J., Hughes, A. M., et al. 2012, ApJ, 744, 162

Appenzeller, I., \& Bertout, C. 2013, A\&A, 558, A83

Ardila, D. R., Herczeg, G. J., Gregory, S. G., et al. 2013, ApJS, 207, 1

Avrett, E. H., \& Loeser, R. 2008, ApJS, 175, 229

Ayres, T. R. 2005, Proc. 13 Cambridge Workshop on Cool Stars, Stellar Systems and the Sun (CoolStars 13), ed. F. Favata, G. A. J. Hussain, \& B. Battrick, ESA SP-560, p. 419

Ayres, T. R. 2010, ApJS, 187, 149 
Basri, G., Johns-Krull, C. M., \& Mathieu, R. D. 1997, AJ, 114, 781

Batalha, C., Batalha, N. M., Alencar, S. H. P., Lopes, D. F., \& Duarte, E. S. 2002, ApJ, 580,343

Bernstein, R. A., Shectman, S. A., Gunnels, S. M., Mochnacki, S., \& Athey, A. E. 2003, SPIE, 4841, 1694

Bertout, C. 1989, ARA\&A, 27, 351

Bouvier, J., Matt, S. P., Mohanty, S., et al. 2014, Protostars and Planets VI, in press (arXiv: 1309.7851)

Brickhouse, N. S., Cranmer, S. R., Dupree, A. K., Luna, G. J. M., \& Wolk, S. 2010, ApJ, 710,1835

Brickhouse, N. S., Cranmer, S. R., Dupree, A. K., et al. 2012, ApJ, 760, L21

Calvet, N., Basri, G., \& Kuhi, L. V. 1984, ApJ, 277, 725

Calvet, N., \& Hartmann, L. 1992, ApJ, 386, 239

Collier Cameron, A., \& Robinson, R. D. 1989, MNRAS, 236, 57

Cranmer, S. R. 2008, ApJ, 680, 316

Cranmer, S. R. 2009, ApJ, 706, 824

Donati, J.-F., Gregory, S. G., Alencar, S. H. P., et al. 2011, MNRAS, 417, 472

Dupree, A. K. 2003, Stars as Suns: Activity, Evolution, and Planets, IAU Symp. 219, A. K. Dupree \& A. O. Benz, eds, p.623

Dupree, A. K., Avrett, E. H., Brickhouse, N. S., Cranmer, S. R., \& Szalai, T. 2008, in Cambridge Workshop on Cool Stars, Stellar Systems and the Sun (CoolStars 14), ed. G. T. VanBelle, ASPCS 384, CD (arXiv:astro-ph/0702395)

Dupree, A. K., Brickhouse, N. S., Cranmer, S. R., et al., 2012, ApJ, 750, 73

Dupree, A. K., Brickhouse, N. S., Smith, G. H., \& Strader, J. 2005a, ApJ, 625, L131

Dupree, A. K., Lobel, A., Young, P. R., et al. 2005b, ApJ, 622, 629

Edwards, S., Fischer, W., Hillenbrand, L. \& Kwan, J. 2006, ApJ, 646, 319. 
Edwards, S., Fischer, W., Kwan, J., Hillenbrand, L., \& Dupree, A. K. 2003, ApJ, 599, L41

Edwards, S., Hartigan, P., Ghandour, L., \& Andrulis, C. 1994, AJ, 108, 1056

Gahm, G. F., Stempels, H. C., Walter, F. W., Petrov, P. P., \& Herczeg, G. J. 2013, A\&A, 560, A 57

Gahm, G. F., Walter, F. M., Stempels, H. C., Petrov, P. P., \& Herczeg, G. J. 2008, A\&A, 482, L35

Gregory, S. G., Wood, K., \& Jardine, M. 2007, MNRAS, 379, L35

Griesmann, U., \& Kling, R. 2000, ApJ, 536, L113

Guilloteau, S., Dutrey, A., Piétu, V., \& Boehler, Y. 2011, A\&A, 529, A105

Gullbring, E., Petrov, P. P., Ilyin, I., Tuominen, I., Gahm, G. F., \& Loden, K. 1996, A\&A, 314,835

Günther, H. M., Wolter, U., Robrade, J., \& Wolk, S. J. 2013, ApJ, 771, 70

Hartmann, L. 1982, ApJS, 48, 109

Hartmann, L. 1998, Accretion Processes in Star Formation, (New York, NY: Cambridge Univ. Press)

Hartmann, L., Hewett, R., \& Calvet, N. 1994, ApJ, 426, 669

Herczeg, G. J., Linsky, J. L., Valenti, J. A., Johns-Krull, C. M., \& Wood, B. E. 2002, ApJ, 572, 310

Howarth, I. D., Prinja, R. K., \& Massa, D. 1995, ApJ, 452, L65

Huélomo, N., Figueira, P., Bonfils, X., et al. 2008, A\&A, 489, L9

Hummer, D. G., \& Rybicki, G. B. 1968, ApJ, 153, L107

Johns-Krull, C., \& Basri, G. 1997, ApJ, 474, 433

Johns-Krull, C., \& Herczeg, G. J. 2007, ApJ, 655, 345 (JKH)

Johnstone, C. P., Jardine, M., Gregory, S. G., Donati, J.-F., \& Hussain, G. 2014, MNRAS, 437,3202

Kaper, L., Henrichs, H. F., Nichols, J. S., \& Telting, J. H. 1999, A\&A, 344, 231 
Kohl, J. L., Noci, G., Cranmer, S. R., \& Raymond, J. C. 2006, A\&AR, 13, 31

Krist, J. E., Stapelfeldt, K. R., Ménard, F., Padgett, D. L., \& Burrows, C. J. 2000, ApJ, 538,793

Kurosawa, R., \& Romanova, M. M. 2012, MNRAS, 426, 2901

Kurosawa, R., \& Romanova, M. M. 2013, MNRAS, 431, 2673

Lamzin, S. A. 1999, Astron. Lett., 25, 430

Landi, E., Gruesbeck, J. R., Lepri, S. T., Zurbuchen, T. H., \& Fisk, L. A. 2012, ApJ, 761, 48

Lehner, N., Fullerton, A. W., Massa, D., Sembach, K. R., \& Zsargó, J. 2003, ApJ, 589, 526

Lobel, A., \& Dupree, A. K. 2001, ApJ, 558, 815

Mallik, S. V. 1986, MNRAS, 222, 307

Massa, D., Fullerton, A. W., Nichols, J. S., et al. 1995, ApJ, 452, L53

Matsakos, T., Chièze, J.-P., Stehlé, C., et al. 2013, A\&A, 557, A69

Matt, S., \& Pudritz, R. E. 2005, ApJ, 632, L135

Matt, S. P., Pinzón, G., Greene, T. P., \& Pudritz, R. E. 2012, ApJ, 745, 101

McClure, M. K., Calvet, N., Espaillat, C., et al. 2013, ApJ, 769, 73

McLean, I. S., Becklin, E. E., Bendiksen, O., et al. 1998, SPIE, 3354, 566

McLean, I. S., McGovern, M. R., Burgasser, A. J., et al. 2000, SPIE, 4008, 1048

McLean, I. S., McGovern, M. R., Burgasser, A. J., et al. 2003, ApJ, 596, 561

Muzerolle, J., Calvet, N., \& Hartmann, L. 2001, ApJ, 550, 944

Muzerolle, J., Luhman, K. L., Briceño, C., Hartmann, L, \& Calvet, N. 2005, ApJ, 625, 906

Natta, A., Testi, L., Muzerolle, J., Randich, S., Comerón, F., \& Persi, P. 2004, A\&A, 424, 603

Oliveira, J. M., Foing, B. H., vanLoon, J. Th., \& Unruh, Y. C. 2000, A\&A, 362, 615

Pascucci, I., Sterzik, M., Alexander, R. D., et al. 2011, ApJ, 736, 13 
Petrov, P. P., Gullbring, E., Ilyin, I., Gahm, G. F., Tuominen, I., Hackman, T., \& Loden, K. 1996, A\&A, 314, 821

Qi, C., Ho, P. T. P., Wilner, D. J., et al. 2004, ApJ, 616, 111

Reale, F., Orlando, S., Testa, P., et al. 2013, Sci, 341, 251

Rigliaco, E., Pascucci, I., Gorti, U., Edwards, S., \& Hollenbach, D. 2013, ApJ, 772:60

Robinson, R. D., \& Carpenter, K. G. 1995, ApJ, 442, 328

Rosenfeld, K. A., Qi, C., Andrews, S. M., et al. 2012, ApJ, 757, 129

Rutten, R. J., \& Uitenbroek, H. 1991, Sol. Phys., 134, 15

Sacco, G. G., Orlando, S., Argiroffi, C., et al. 2010, A\&A, 522, A55

Schegerer, A. A., Wolf, S., Hummel, C. A., Quanz, S. P., \& Richichi, A. 2009, A\&A, 502, 367

Skelly, M. B., Unruh, Y. C., Collier Cameron, A., et al. 2008, MNRAS, 385, 708

Tripathi, D., Mason, H. E., \& Klimchuk, J. A. 2010, ApJ, 723, 713

Vacca, W. D. \& Sandell, G. 2011, ApJ, 732, 8

White, R. J., \& Basri, G. 2003, ApJ, 582, 1109

Wichmann, R., Bastian, U., Krautter, J., Jankovics, I., \& Rucinski, S. M. 1998, MNRAS, 301, L39

Yang, J., Herczeg, G. J., Linsky, J. L., et al. 2012, ApJ, 744, 121 

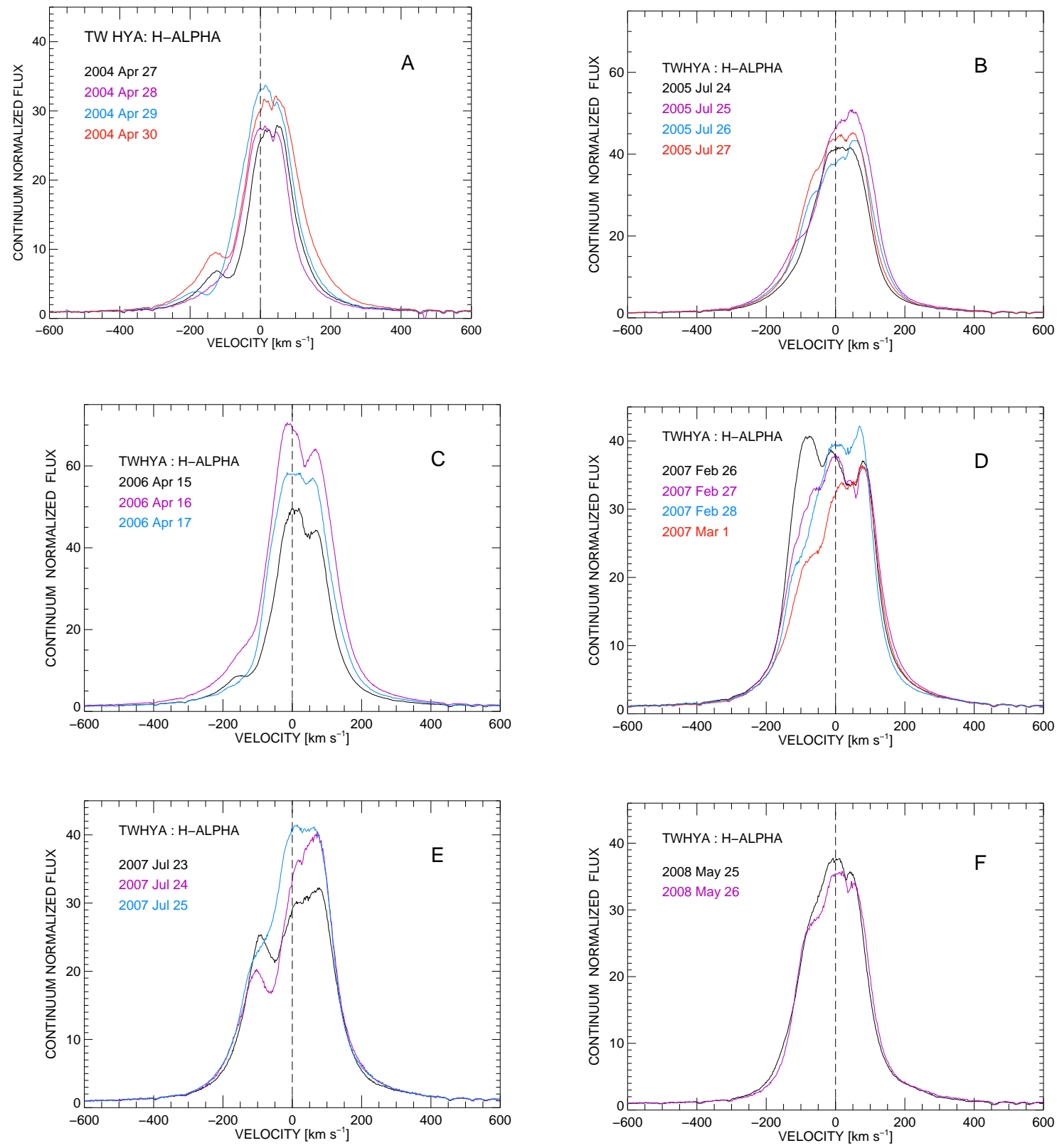

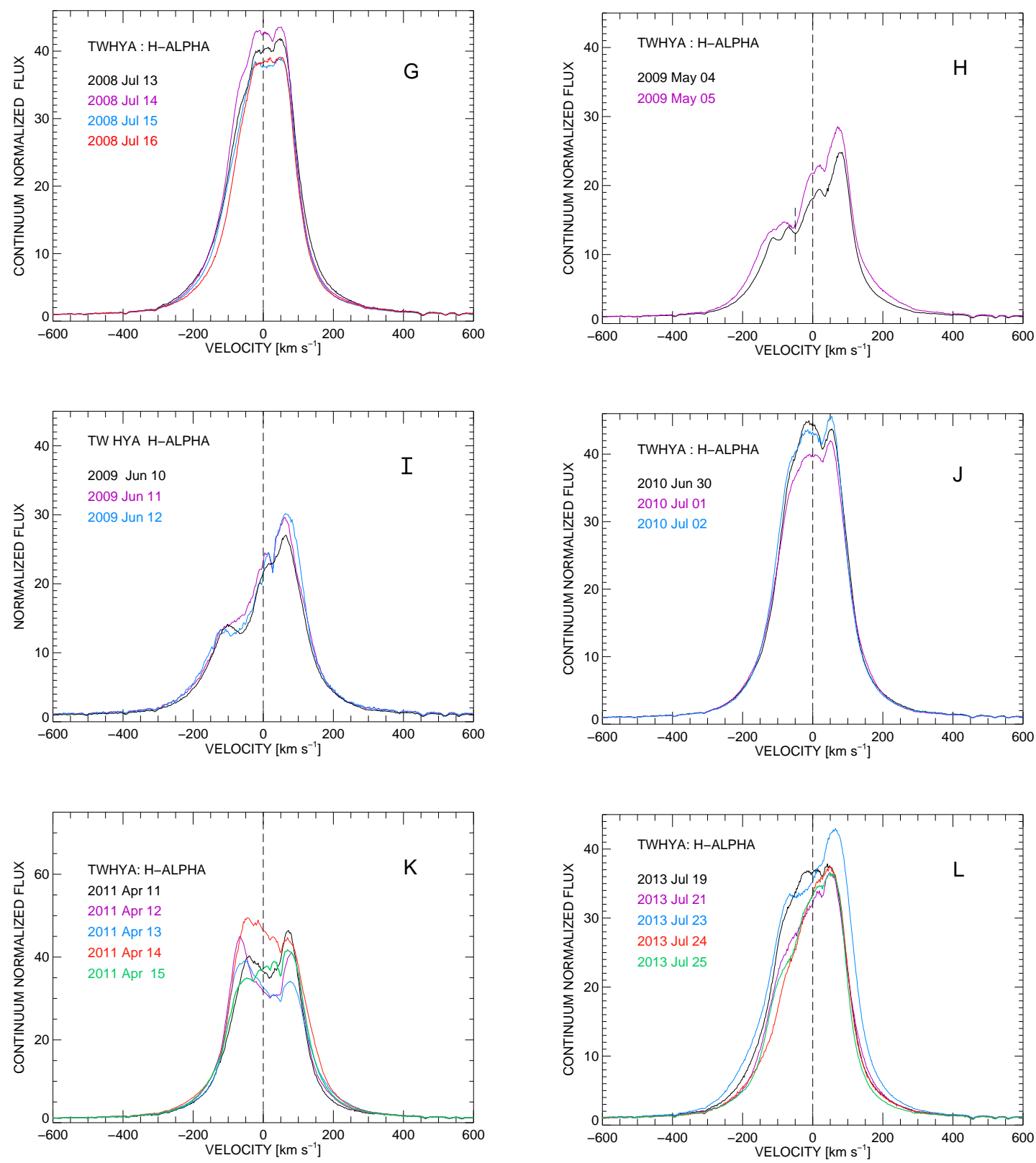

Fig. 1. - Single H $\alpha$ spectra of TW Hya selected from ten years of measurements from 2004 to 2013. Note that there are two different scales on the y-axis. Observations in 2005 Jul, 2006 Apr, and 2011 Apr indicate stronger $\mathrm{H} \alpha$. The narrow absorption feature near $+50 \mathrm{~km} \mathrm{~s}^{-1}$ is due to water vapor. All of these spectra suggest that $\mathrm{H} \alpha$ is centered on the stellar radial velocity, and the profile is substantially modified by wind absorption causing the apparent 'red' enhancement. 


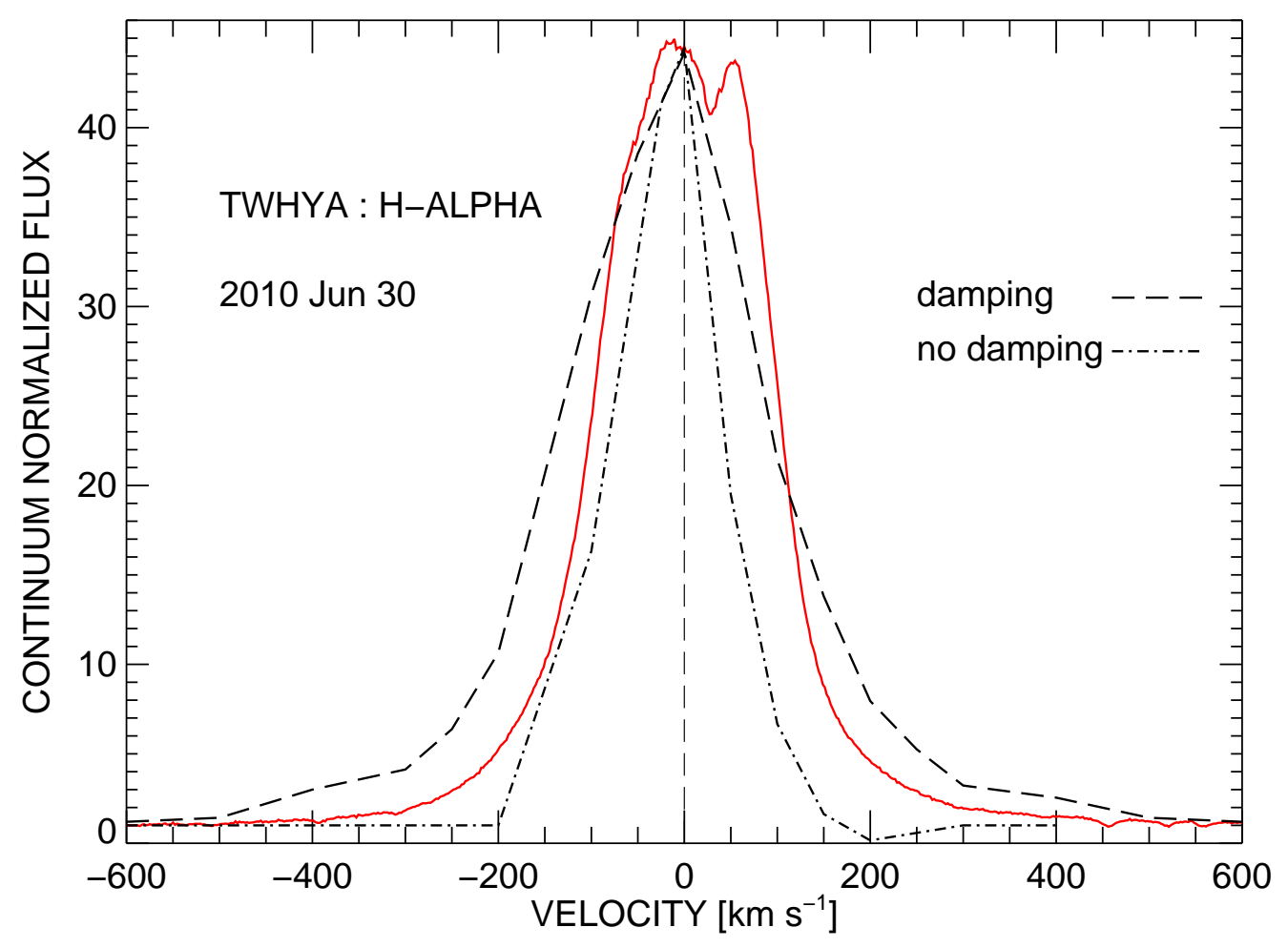

Fig. 2.- The observed $\mathrm{H} \alpha$ spectrum from 2010 Jun 30 (solid line) with two models overlaid from Muzzerolle et al. (2001) that assume either no line-damping or an arbitrary damping parameter. These models assume formation of $\mathrm{H} \alpha$ in an accretion stream which can be seen by the weaker positive velocity side of the profile as compared with the negative side, and slight subcontinuum absorption when damping is absent. A stellar wind is not included in the models accounting in part for the excess emission of the model on the negative velocity side. Also the model profiles appear 'pointed' which is not found in the observed profiles (see also Figure 1). 


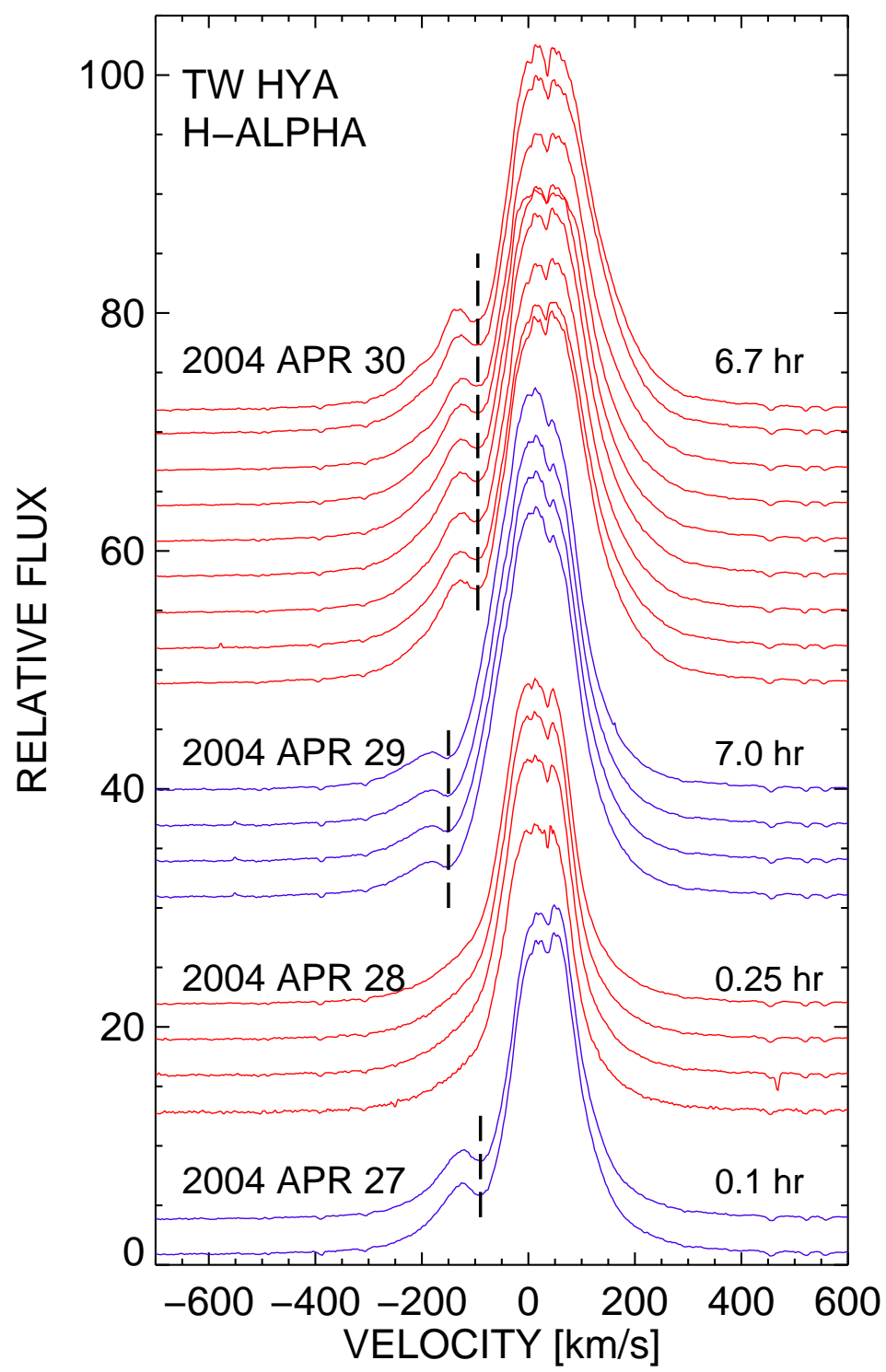

Fig. 3.- Magellan/MIKE spectra over 4 nights in 2004 showing the appearance of wind structures stable in velocity. The time interval between the first and last spectrum is shown. Each spectrum has been offset for display. The broken vertical lines mark the constant velocity of the absorption feature during each night. The notch in the $\mathrm{H} \alpha$ emission at +50 $\mathrm{km} \mathrm{s}^{-1}$ results from water vapor absorption. 


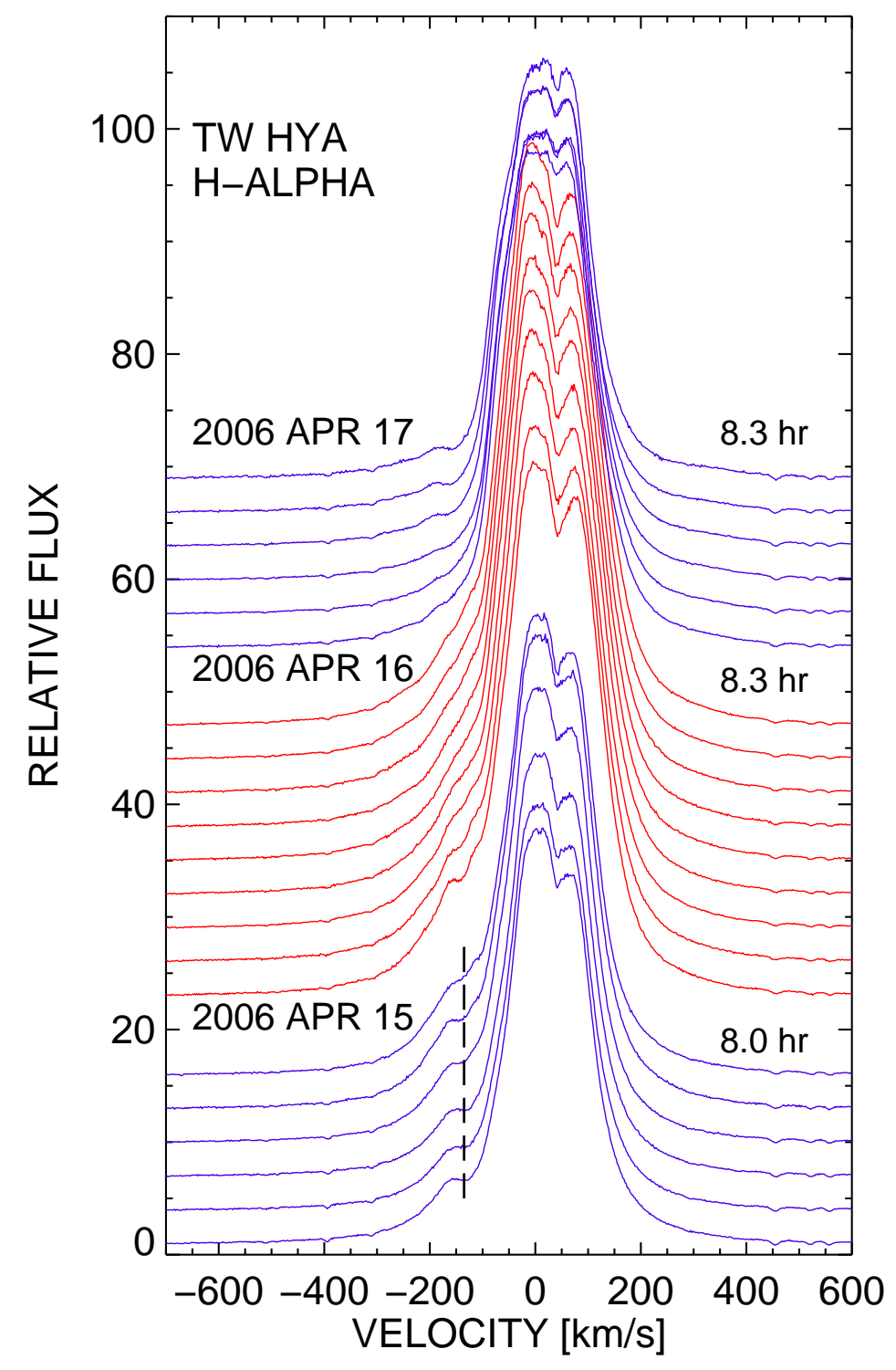

Fig. 4.- Magellan/MIKE spectra of the $\mathrm{H} \alpha$ line over 3 consecutive nights in April 2006. The narrow absorption near $+50 \mathrm{~km} \mathrm{~s}^{-1}$ is caused by water vapor. More spectra were obtained than displayed here and a gray scale representation of all spectra is shown in Figure 5. 

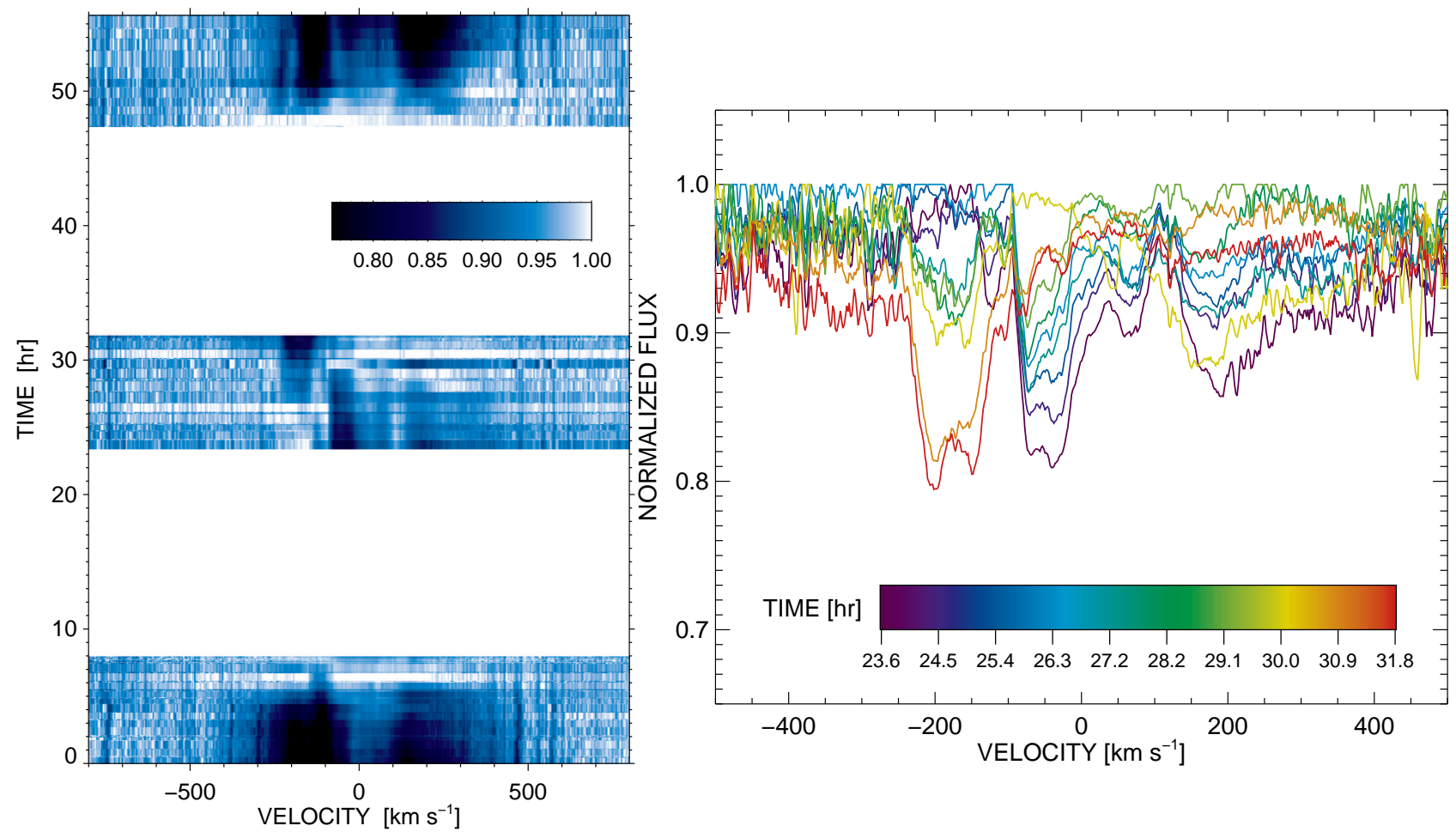

Fig. 5.- Left panel: Gray scale representation of the $\mathrm{H} \alpha$ profiles of TW Hya over 3 consecutive nights in April 2006 where each spectrum has been divided by the maximum flux at each wavelength in order to display absorption features. During Night 2, absorption at -50 $\mathrm{km} \mathrm{s}^{-1}$ is replaced by a discontinuous jump to a new absorption feature at $-200 \mathrm{~km} \mathrm{~s}^{-1}$ (at $\sim 29$ hrs). Right panel: Profiles during night 2 of the gray scale representation above. Note the weakening of the absorption at $-50 \mathrm{~km} \mathrm{~s}^{-1}$ during the night, and the abrupt appearance of another absorption feature at $-200 \mathrm{~km} \mathrm{~s}^{-1}$ in the middle of the night which becomes prominent at the end of the night. Broad absorption extending from $\sim+150$ to $\sim+400$ $\mathrm{km} \mathrm{s}^{-1}$ arising from infalling material is variable, and the velocities are commensurate with the velocities measured in He I. 

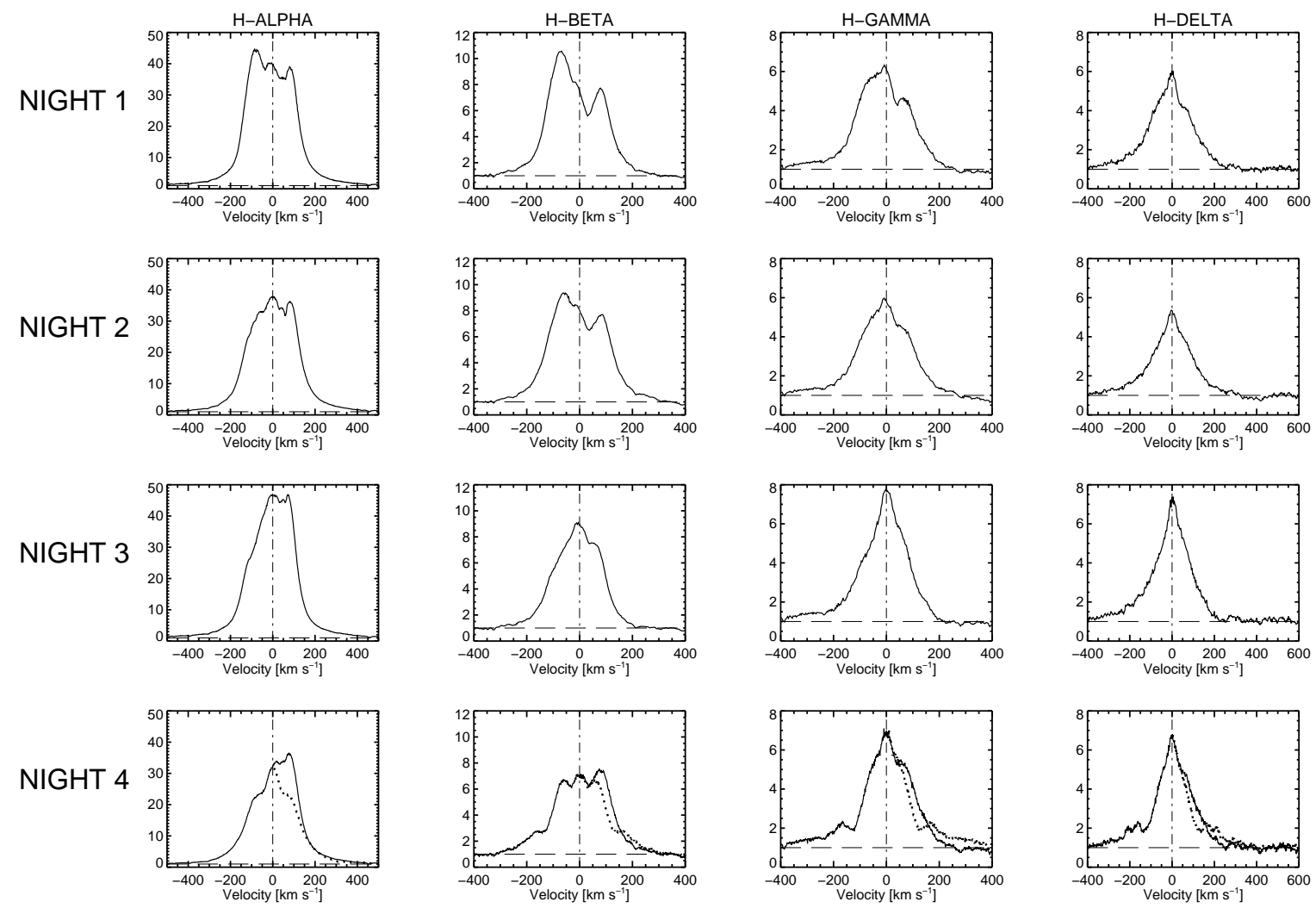

Fig. 6.- Balmer series over 4 consecutive nights in 2007 (Feb. 26 - Mar. 1). The y-axis represents a continuum normalized flux. Profiles from night 4 (2007 March 1) have the negative velocity segments overlaid on the positive velocity side and marked by a broken (red) line. The effects of wind absorption can be seen when the broken line lies below the solid line; effects of absorption by the accretion stream can be seen at $\sim+200 \mathrm{~km} \mathrm{~s}^{-1}$ where the broken line lies slightly above the solid line. 


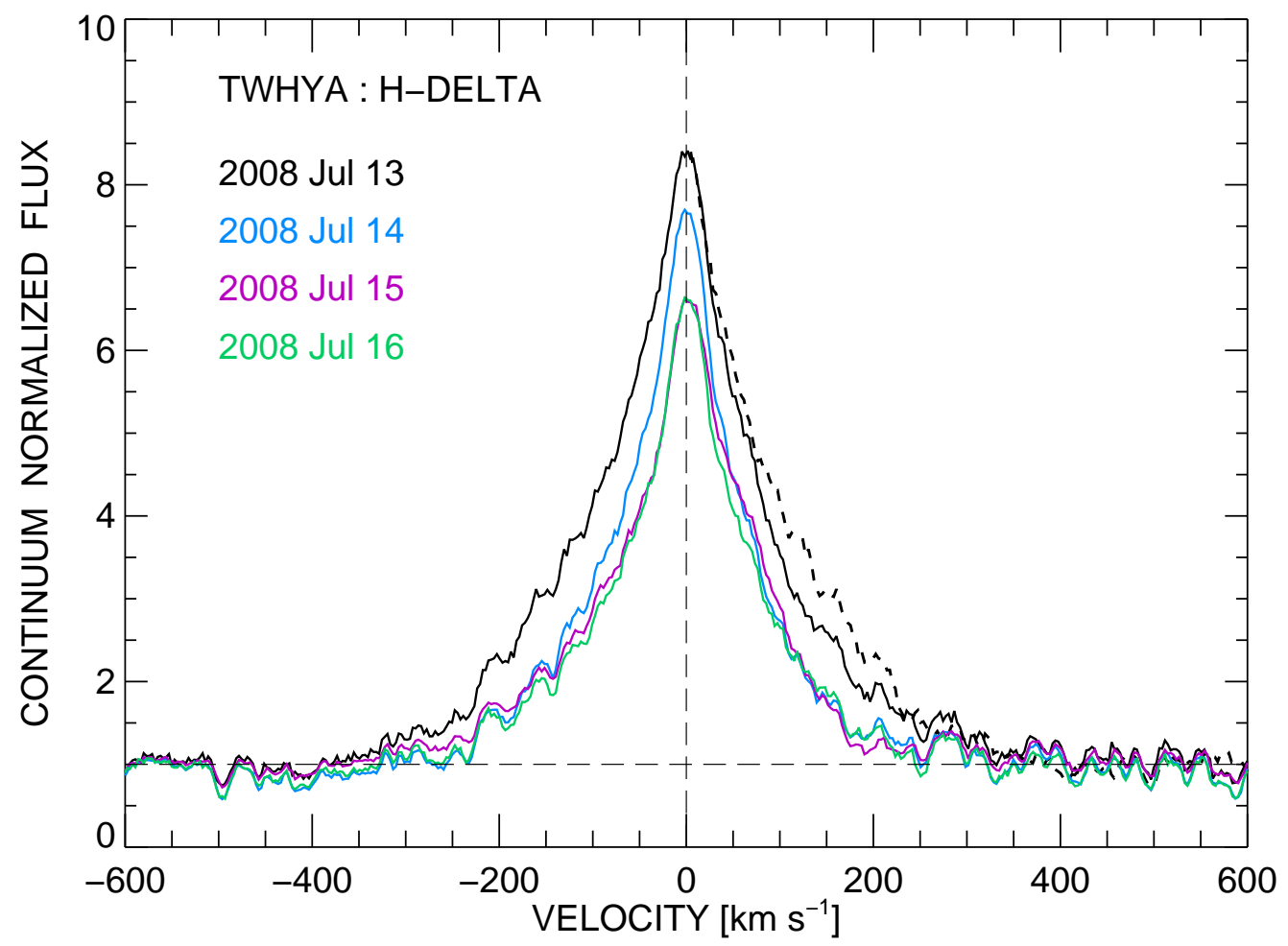

Fig. 7.- Typical H $\delta$ line profile $(\lambda 4101.73)$ over 4 consecutive nights in 2008. The broken line represents the negative velocity wing of the first night's observation that has been reflected around the axis at zero velocity. This illustrates the slight broad absorption present at velocities +50 to $+250 \mathrm{~km} \mathrm{~s}^{-1}$. Profiles from the following 3 nights are symmetric. Subcontinuum absorption predicted by magnetospheric accretion models is missing in the positive velocity wing of the line. 


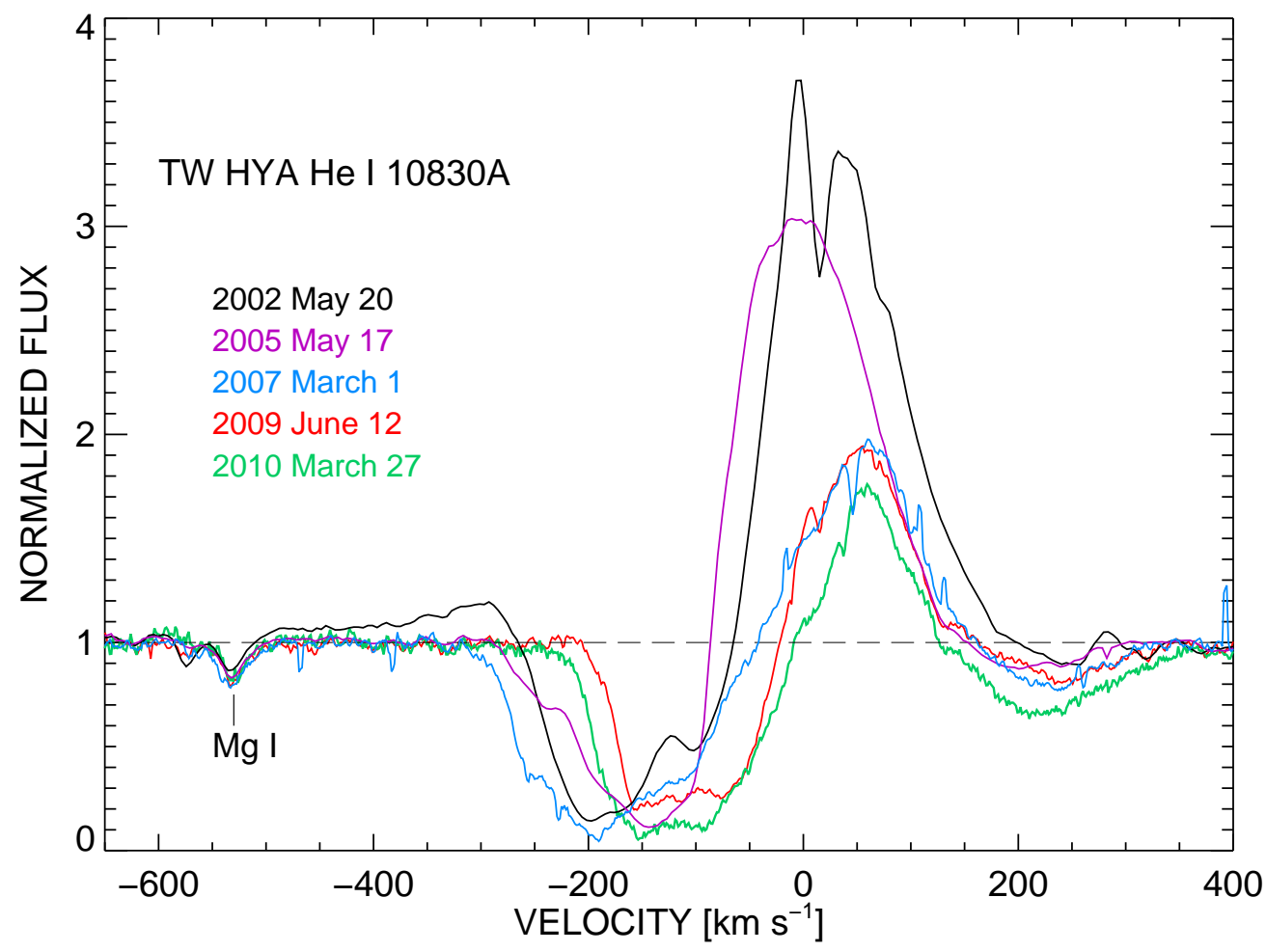

Fig. 8. - Spectra of He I $10830 \AA$ spanning 8 years. Substantial changes in the emission flux level, the wind opacity, the wind speed, and the inflowing absorption are evident. 

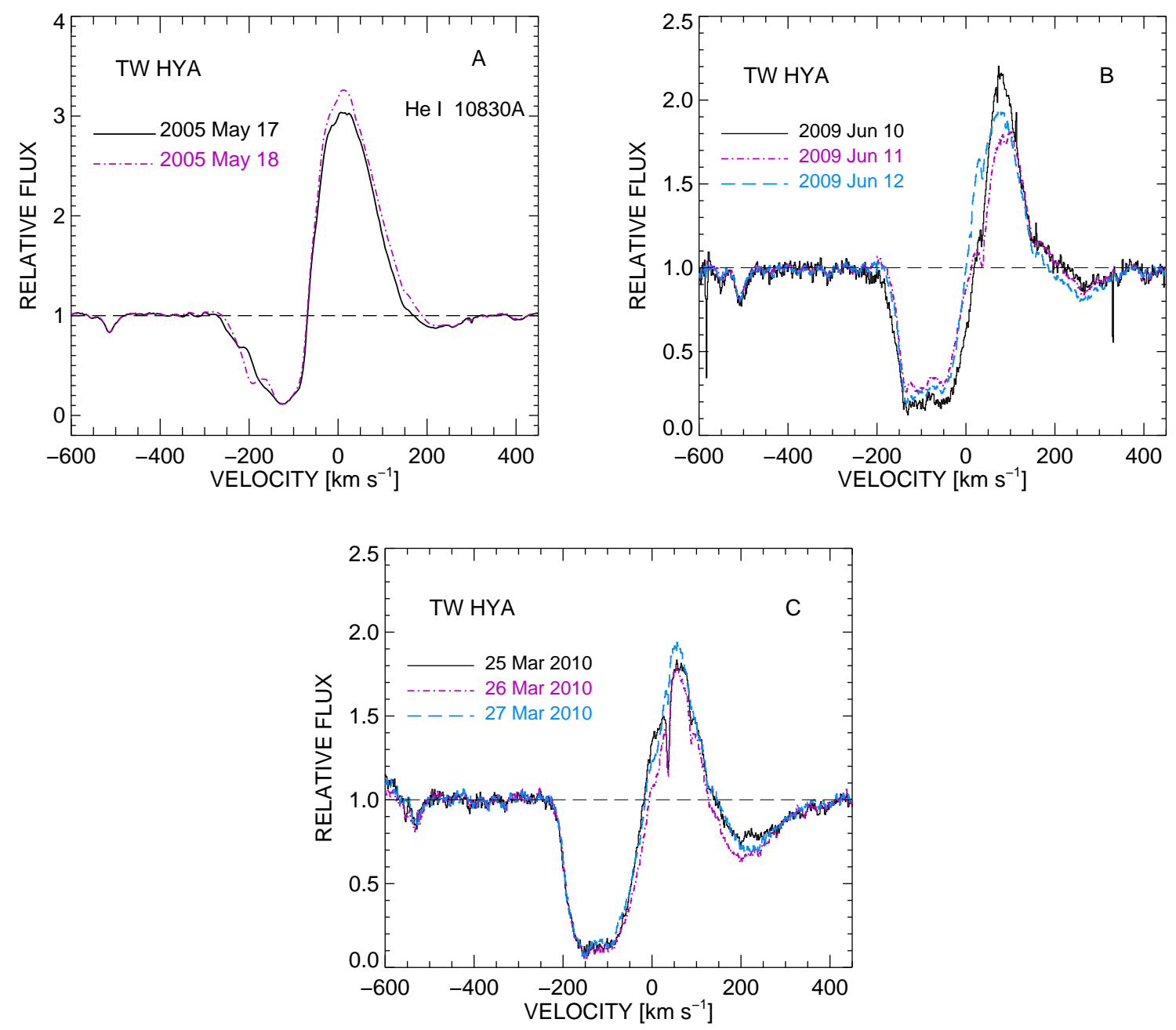

Fig. 9.- KECK II NIRSPEC spectra (2005) and Gemini-S PHOENIX spectra (2009, 2010) of TW Hya on successive nights. The outflowing wind remains relatively stable as compared to both the emission and the inflowing material. 

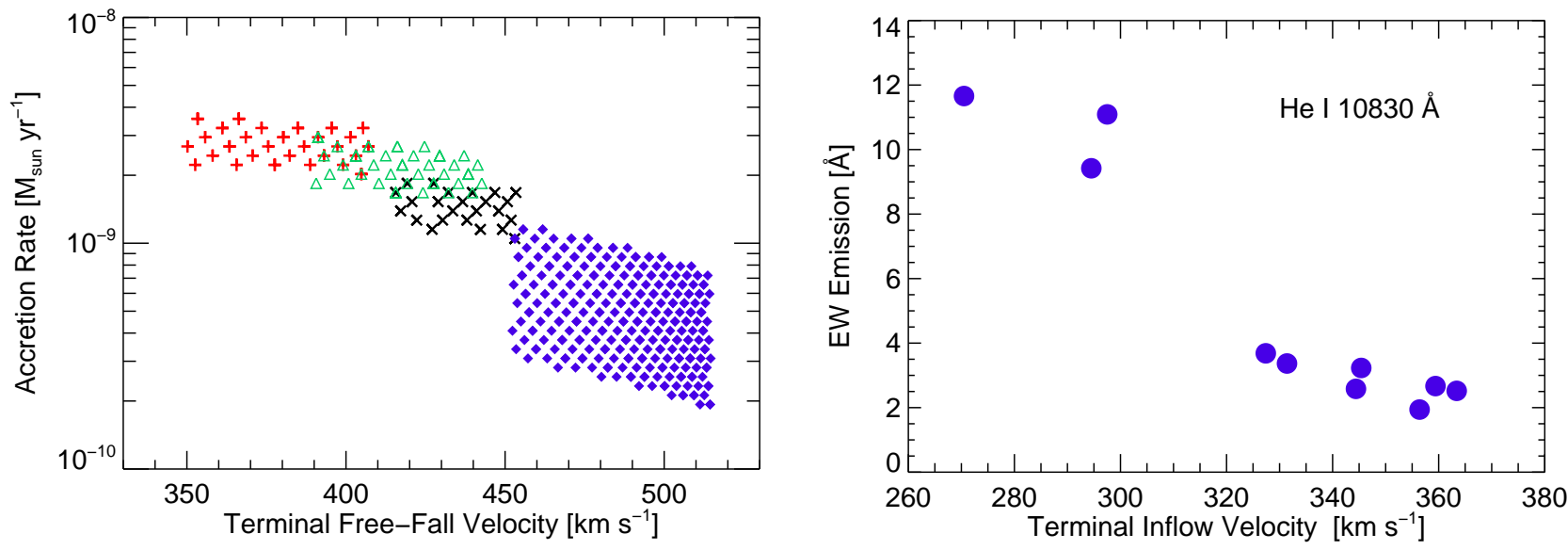

Fig. 10.- Left panel: The magnetic dipole accretion model predicts the relationship between the accretion rate and the terminal free-fall velocity. A slower infall velocity results from a smaller radius of the inner circumstellar disk, and a larger filling factor in the stellar atmosphere produces a higher mass accretion rate. Parameter ranges constrained by CHANDRA Ne IX diagnostics are shown here. The $\times$ symbols denote the average CHANDRA spectrum, while the other symbols mark 3 individual CHANDRA pointings (cf. Brickhouse et al. 2012). Right panel: Equivalent width of the emission component of the He I line $(\lambda 10830)$ as a function of the terminal velocity indicated by the inflowing subcontinuum absorption. These observations display general agreement with the magnetic dipole accretion model. The emission in the post-shock cooling zone increases because of increased accretion at lower terminal free-fall velocities. 


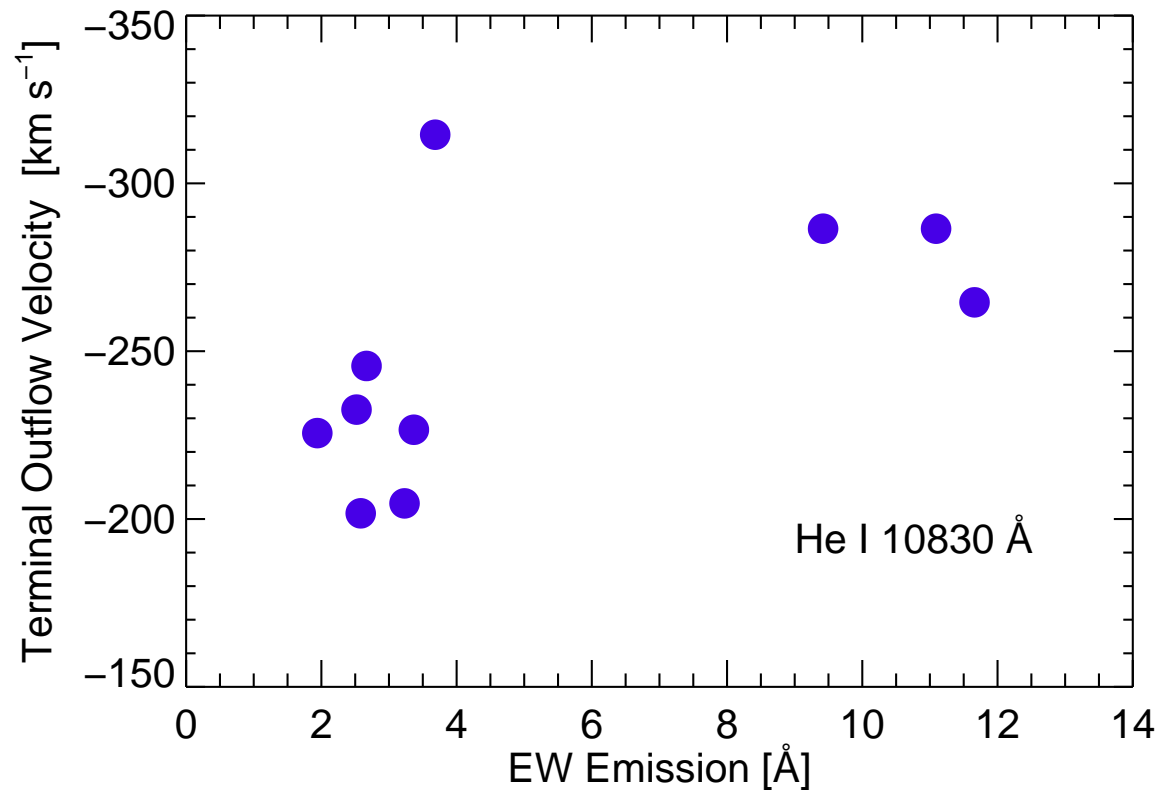

Fig. 11. - The terminal outflow velocity as a function of the emission component of the He I. Except for one outlier (2007 Mar. 1), fast outflow is correlated with increased emission. This is not inconsistent with the conclusion that the turbulent post-shock region can affect the wind speed. 

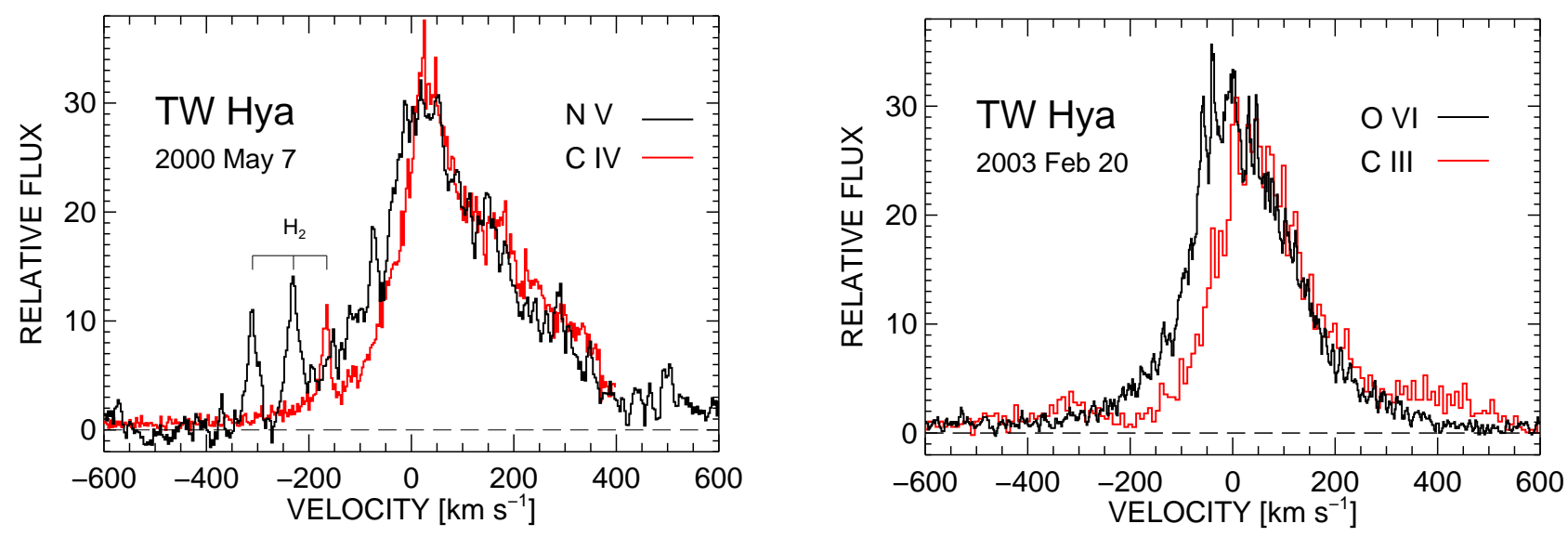

Fig. 12.- UV and far-UV spectra of major resonance lines. Peak fluxes are scaled to match to illustrate the shape of the profiles. Left panel: HST:STIS spectra of N V $(\lambda 1239)$ and C IV $(\lambda 1548)$ taken in the year 2000. Right panel: FUSE spectra of C III $(\lambda 977)$ and O VI taken three years later in 2003 (Dupree et al. 2005a). Note that the profiles observed at the same time are similar one to another with the exception of different absorption levels on the negative velocity side (see text for explanation).

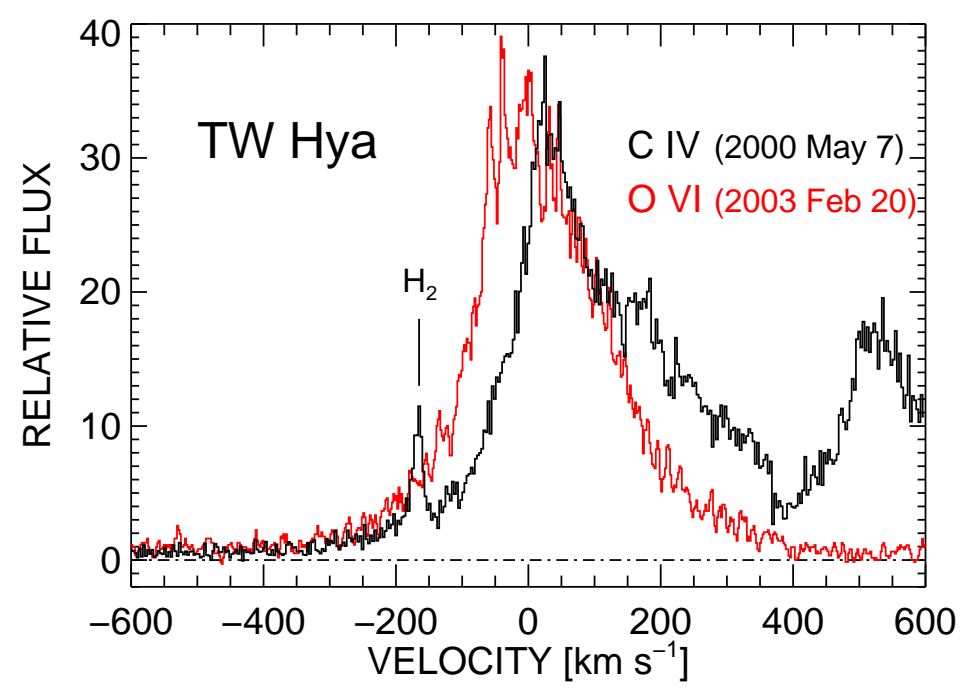

Fig. 13.- Comparison of resonance lines at different times showing the dramatic change in emission at positive velocities. The second member of the C IV doublet appears at +500 $\mathrm{km} \mathrm{s}^{-1}$. See text for discussion of these profiles. Peak fluxes are scaled in this figure to illustrate the shape of the line profiles. 

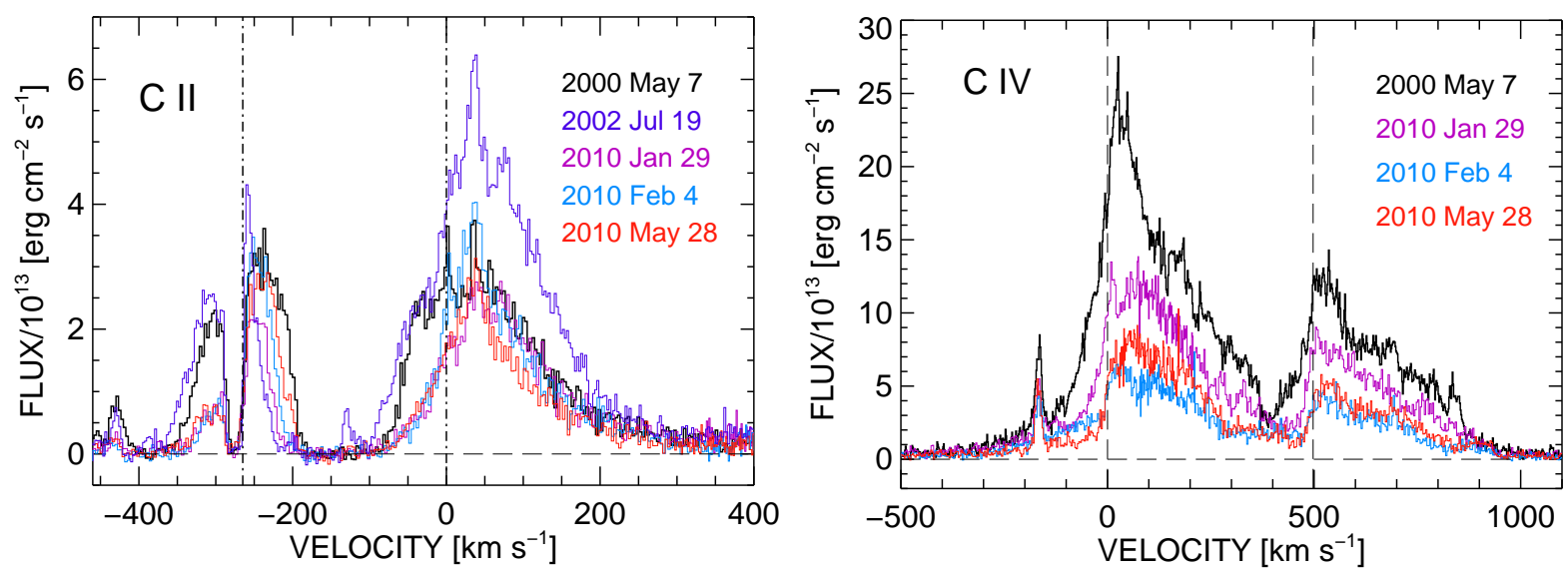

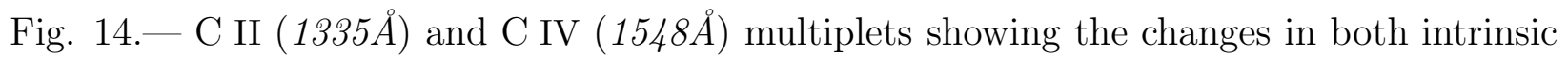
line strength and wind opacity, even on short time scales, such as the 6 days separating 2010 Jan 29 and 2010 Feb 4. The C II line is generally narrower on the positive velocity side than the $\mathrm{C}$ IV which is similar to the high temperature $\mathrm{N}$ V profile. The broken vertical lines mark the rest velocities of the doublets.
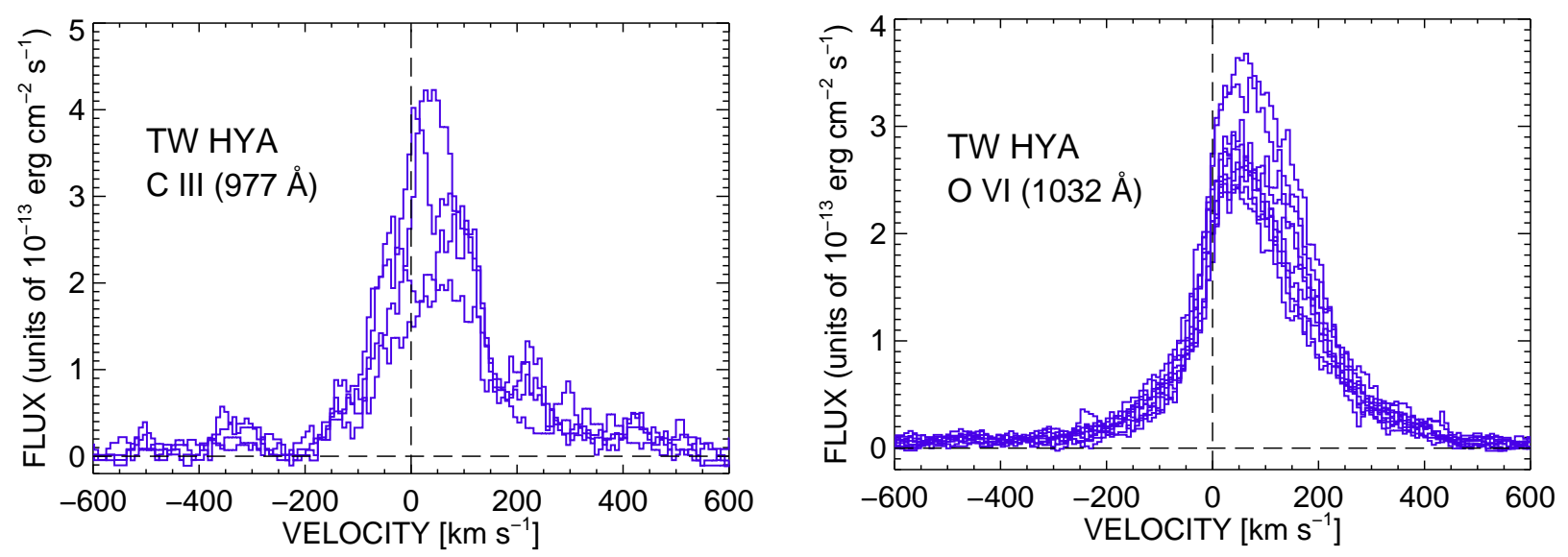

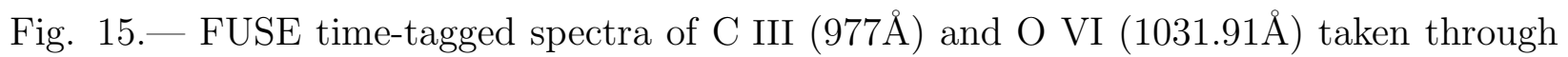
the LWRS aperture from the SiC2A and LiF1A detector measured over a 32.2 hour span 2003 Feb 20-21. The variability at positive velocities is more substantial in both lines than at negative velocities. The opacity of the hot wind as measured in the O VI line remains relatively constant. Flux measurements from the individual spectra are shown in Figure 16. 


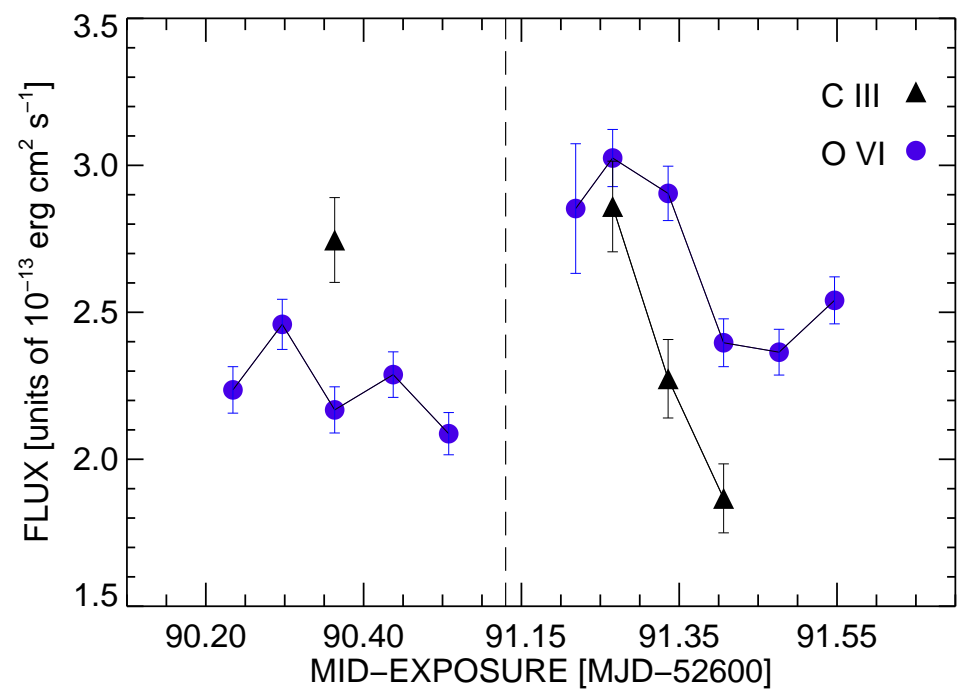

Fig. 16.- Fluxes measured from the FUSE line profiles shown in the previous figure display similar behavior in the C III and O VI emission during several hours of the second pointing. During the second pointing, the absolute flux values are reversed from the first pointing, and the line fluxes are correlated. 

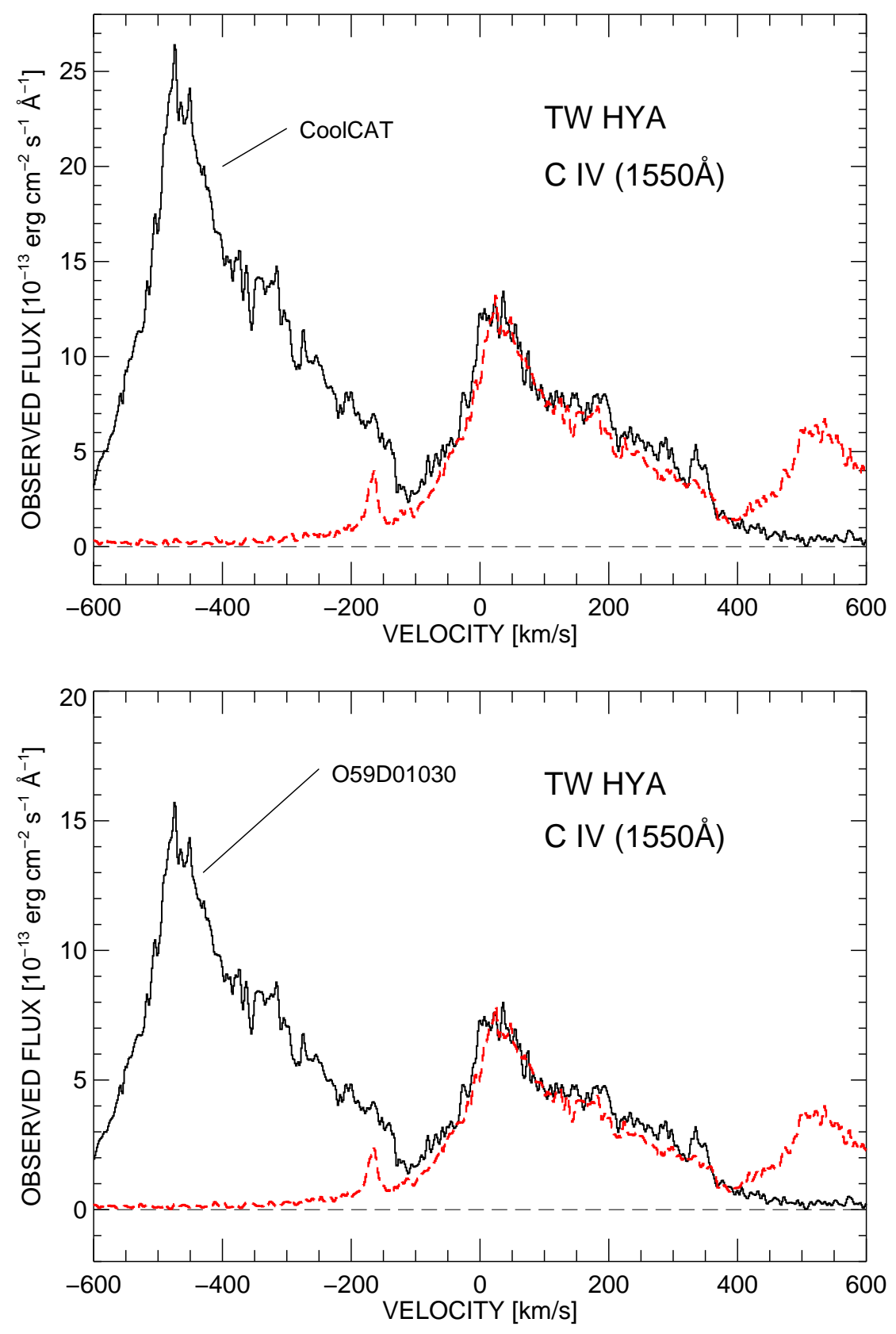

Fig. 17. - The solid lines show the 'CoolCAT' optimum reduction (upper panel) of the same STIS spectrum as that used by JKH (lower panel, O59D01030). The broken lines (red in online version) represent the profile of the $1548 \AA$ multiplet multiplied by 0.5 and shifted by the velocity equivalent of the wavelength separation of the doublet. Wind absorption by the $1550 \AA$ component is indicated by the weaker extended positive velocity wing of the $1548 \AA$ transition (broken line). Wind absorption occurs systematically between +175 and +375 $\mathrm{km} \mathrm{s}^{-1}$ of the scaled $1548 \AA$ line in the velocity scale of the figure. Additionally the effect of increased opacity in the $1548 \AA$ line is evident in the region -125 to $+25 \mathrm{~km} \mathrm{~s}^{-1}$, where the broken line lies systematically below the solid line. Thus, two signatures of wind absorption can be noted in these doublet profiles: absorption of the red wing of $1548 \AA$ by the blue wing of $1550 \AA$, and the increased scattering of the $1548 \AA$ line in the wind as compared with $1550 \AA$. 


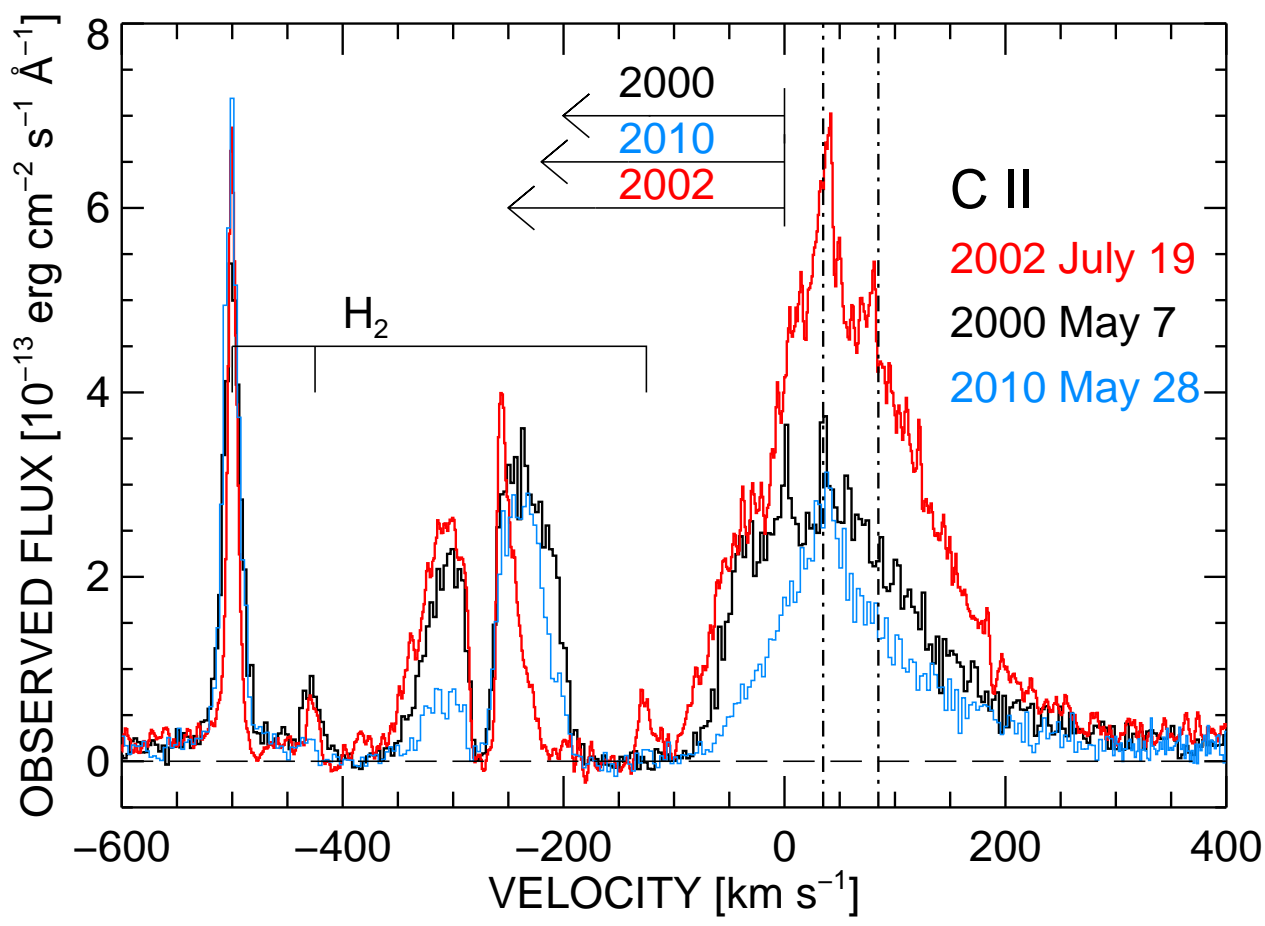

Fig. 18. - HST/STIS spectra of TW Hya in the region of the C II $1335 \AA$ doublet in 2000, 2002, and 2010 showing the variabilities in C II emission, $\mathrm{H}_{2}$ emission and wind speed and opacity. The short wavelength component $\left(-275 \mathrm{~km} \mathrm{~s}^{-1}\right)$ of the doublet is bifurcated by interstellar absorption. The broken vertical lines mark the region $\left(+35\right.$ to $\left.+85 \mathrm{~km} \mathrm{~s}^{-1}\right)$ where JKH postulated the lack of absorption. This section of the profile is obviously substantially variable in both flux and slope of the emission line. The arrows indicate the extent of wind absorption for one member of the doublet. Note that the $H_{2}$ lines vary, but not in any systematic way with wind speed or opacity. In 2002, when the wind opacity is substantial between -100 and $-250 \mathrm{~km} \mathrm{~s}^{-1}$, another $H_{2}$ emission line appears $(\mathrm{R}(2) 0-4$ at $1335.2 \AA$ at $\left.\sim-125 \mathrm{~km} \mathrm{~s}^{-1}\right)$. 
Table 1. TW Hya Observations

\begin{tabular}{|c|c|c|c|c|}
\hline $\begin{array}{l}\text { Spectral } \\
\text { Region }\end{array}$ & Instrument & Date & $\begin{array}{c}\text { Data file/ } \\
\text { spectra }\end{array}$ & $\begin{array}{c}\text { Exp. time } \\
(\mathrm{s})\end{array}$ \\
\hline \multirow[t]{2}{*}{ Far UV } & FUSE & 2003 Feb 20 & $\mathrm{C} 0670101^{\mathrm{a}}$ & 15221 \\
\hline & FUSE & 2003 Feb 21 & $\mathrm{C} 0670102^{\mathrm{a}}$ & 15465 \\
\hline \multirow[t]{5}{*}{ UV } & HST/STIS/E140M & 2000 May 7 & O59D01030 & 2300 \\
\hline & HST/STIS/E140H & 2002 July 19 & StarCAT $^{c}$ & 4355 \\
\hline & HST/STIS/E140M & 2010 Jan 29 & OB3R07060 & 3058 \\
\hline & HST/STIS/E140M & 2010 Feb 04 & OB3R08060 & 3058 \\
\hline & HST/STIS/E140M & 2010 May 28 & OB3R09060 & 3058 \\
\hline \multirow[t]{12}{*}{ Optical } & Magellan/CLAY/MIKE & 2004 Apr $27-30$ & 19 & $60-300$ \\
\hline & Magellan/CLAY/MIKE & 2005 Jul 24-27 & 4 & $60-300$ \\
\hline & Magellan/CLAY/MIKE & 2006 Apr $15-17$ & 19 & $60-300$ \\
\hline & Magellan/CLAY/MIKE & 2007 Feb 26-Mar 1 & $\sim 360$ & $45-360$ \\
\hline & Magellan/CLAY/MIKE & 2007 Jul 23-25 & 4 & $45-60$ \\
\hline & Magellan/CLAY/MIKE & 2008 May 25-26 & 7 & $45-240$ \\
\hline & Magellan/CLAY/MIKE & 2008 Jul 13-16 & 9 & $35-180$ \\
\hline & Magellan/CLAY/MIKE & 2009 May 4-5 & 5 & $45-180$ \\
\hline & FLWO/TRES & 2009 Jun 10-12 & 3 & 720 \\
\hline & Magellan/CLAY/MIKE & 2010 Jun 30-Jul 2 & 3 & 45 \\
\hline & FLWO/TRES & 2011 Apr 11-15 & 5 & 1200 \\
\hline & Magellan/CLAY/MIKE & 2013 Jul 19-25 & 5 & $30-180$ \\
\hline \multirow[t]{5}{*}{ Near IR } & KECK II/NIRSPEC & 2002 May 20 & 2 & 200 \\
\hline & KECK II/NIRSPEC & 2005 May $17-18$ & 4 & 200 \\
\hline & Gemini-S/PHOENIX & 2007 Mar 1 & 1 & 900 \\
\hline & Gemini-S/PHOENIX & 2009 Jun $10-12$ & 3 & 300 \\
\hline & Gemini-S/PHOENIX & 2010 Mar 25-27 & 3 & 300 \\
\hline
\end{tabular}

aPI: J. Linsky

${ }^{\mathrm{b}}$ CoolCAT (casa.colorado.edu/ ayres/CoolCAT) was derived from this spectrum.

'StarCAT can be found at archive.stsci.edu/prepds/starcat (Ayres 2010). 
${ }^{d}$ PI: N. Calvet 